\title{
BCAS1 expression defines a population of early myelinating oligodendrocytes in multiple sclerosis lesions
}

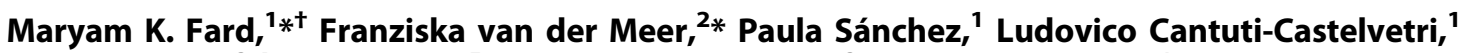 \\ Sunit Mandad, ${ }^{3,4}$ Sarah Jäkel, ${ }^{5}$ Eugenio F. Fornasiero, ${ }^{3}$ Sebastian Schmitt, ${ }^{1}$ \\ Marc Ehrlich, ${ }^{6,7}$ Laura Starost, ${ }^{6,7}$ Tanja KuhImann, ${ }^{7}$ Christina Sergiou, ${ }^{2}$ Verena Schultz, ${ }^{2}$ \\ Claudia Wrzos, ${ }^{2}$ Wolfgang Brück, ${ }^{2}$ Henning Urlaub, ${ }^{4,8}$ Leda Dimou, ${ }^{5,9,10}$ \\ Christine Stadelmann, ${ }^{2 \dagger \neq}$ Mikael Simons ${ }^{1,10,11,12 † \neq}$
}

Investigations into brain function and disease depend on the precise classification of neural cell types. Cells of the oligodendrocyte lineage differ greatly in their morphology, but accurate identification has thus far only been possible for oligodendrocyte progenitor cells and mature oligodendrocytes in humans. We find that breast carcinoma amplified sequence 1 (BCAS1) expression identifies an oligodendroglial subpopulation in the mouse and human brain. These cells are newly formed, myelinating oligodendrocytes that segregate from oligodendrocyte progenitor cells and mature oligodendrocytes and mark regions of active myelin formation in development and in the adult. We find that $\mathrm{BCAS}^{+}$oligodendrocytes are restricted to the fetal and early postnatal human white matter but remain in the cortical gray matter until old age. $\mathrm{BCAS}^{+}$oligodendrocytes are reformed after experimental demyelination and found in a proportion of chronic white matter lesions of patients with multiple sclerosis (MS) even in a subset of patients with advanced disease. Our work identifies a means to map ongoing myelin formation in health and disease and presents a potential cellular target for remyelination therapies in MS.

\section{INTRODUCTION}

Oligodendrocytes wrap several layers of their plasma membrane around axons to generate myelin (1), which provides electrical insulation to increase the speed of nerve conduction (2). Oligodendrocyte and myelin development progresses along a typical chronological and topographic sequence, starting in areas related to basic homeostasis and progressing to regions controlling more complex functions (3). However, in mice, myelin formation is not only a developmental process driven by a predefined intrinsic program but also extends into adulthood where it is regulated by environmental factors (4-6). The developing and adult mouse central nervous system (CNS) both contain an abundant population of oligodendrocyte progenitor cells that continuously generate oligodendrocytes (7) and provide a source for reforming myelin after injury (8). Both oligodendrocyte progenitor cell proliferation and differentiation can increase within active neuronal circuits, and there is evidence that adult-born oligodendrocytes are actively engaged in forming

\footnotetext{
${ }^{1}$ Max Planck Institute for Experimental Medicine, 37075 Göttingen, Germany. ${ }^{2}$ Department of Neuropathology, University of Göttingen Medical Center, 37075 Göttingen, Germany. ${ }^{3}$ Department of Neuro- and Sensory Physiology, University of Göttingen Medical Center, 37073 Göttingen, Germany. ${ }^{4}$ Max Planck Institute for Biophysical Chemistry, 37073 Göttingen, Germany. ${ }^{5}$ Department of Physiological Genomics, BioMedical Center, Ludwig-Maximilians-Universität München, Munich, Germany. ${ }^{6}$ Institute of Neuropathology, University Hospital Münster, 48149 Münster, Germany. ${ }^{7}$ Department of Cell and Developmental Biology, Max Planck Institute for Molecular Biomedicine, 48149 Münster, Germany. ${ }^{8}$ Bioanalytical Mass Spectrometry Group, Department of Clinical Chemistry, University of Göttingen Medical Center, Robert Koch Straße 40, 37075 Göttingen, Germany. ${ }^{9}$ Molecular and Translational Neuroscience, Department of Neurology, Medical Faculty, Ulm University, 89081 Ulm, Germany.

${ }^{10}$ Munich Cluster for Systems Neurology (SyNergy), 81377 Munich, Germany. ${ }^{11}$ Institute of Neuronal Cell Biology, Technical University of Munich, 80805 Munich, Germany.

${ }^{12}$ German Center for Neurodegenerative Diseases (DZNE), 6250 Munich, Germany.

*These authors contributed equally to this work.

+Corresponding author. Email: cstadelmann@med.uni-goettingen.de (C.S.);

khojasteh@em.mpg.de (M.K.F.); msimons@gwdg.de (M.S.)

¥These authors contributed equally to this work as co-senior authors.
}

new myelin sheaths in mice (9-11). For example, in adult mice, learning a new motor task is associated with changes in white matter structure (12). In humans, a large number of studies have demonstrated white matter changes upon learning and training, such as extensive piano playing (13), suggesting de novo myelination upon neuronal stimulation (14). However, human studies are often based on magnetic resonance imaging, which does not provide direct information on myelin; therefore, whether and how brain activity modulates myelin changes is not well understood. Birth dating of oligodendrocytes, by analysis of the integration of nuclear bomb test-derived ${ }^{14} \mathrm{C}$ into cells, provided evidence for the generation of only few new oligodendrocytes in the adult human corpus callosum (15), suggesting that adaptive myelination in the white matter of humans may be more limited as compared to mice. Evidence for the de novo formation of myelin in adults comes from studies on human demyelinating diseases, of which multiple sclerosis (MS) is the most prevalent. On average, in MS, only $20 \%$ of patients display efficient (above 60\%) remyelination of lesions as shown by histochemistry in areas of pale myelin stain called "shadow plaques" (16). Currently, no molecular marker exists that allows the discrimination of myelinated from remyelinated axons, and ongoing myelination is notoriously difficult to detect; consequently, it has thus far not been possible to determine whether new myelin sheaths are formed in chronically demyelinated MS lesions. Because failure of remyelination contributes to disease progression in MS, remyelinating oligodendrocytes might represent an important drug target. Here, we set out to develop a new method to visualize actively myelinating oligodendrocytes in mouse and human to map areas of active myelination in health and disease. This led to the identification of breast carcinoma amplified sequence 1 (BCAS1) as a marker for ongoing myelination and remyelination. BCAS1 was originally identified as mRNA amplified in human cancer cell lines (17), but recent transcriptome studies have shown that it is also highly expressed in the brain and, more specifically, in oligodendrocytes (18). The 
function of BCAS1 is unknown, but there is evidence that it interacts with the dynein light chain (19) and is required for brain function because its absence results in reduced anxiety and schizophrenia-like behavioral abnormalities in mice (20).

We found that $\mathrm{BCAS1}^{+}$cells represent a population of oligodendrocytes that segregates from mature oligodendrocytes and their progenitors in humans. Our results show that in humans, the high density of $\mathrm{BCAS}^{+}$oligodendrocytes is only found in the white matter in the fetal and early postnatal period, whereas in the frontal cortex, oligodendrocytes expressing BCAS1 are numerous throughout adulthood. We observed $\mathrm{BCAS1}^{+}$oligodendrocytes at the lesion edge in a proportion of fully demyelinated white matter lesions in MS patients, suggesting that endogenous remyelination might be active during MS even at late stages.

\section{RESULTS \\ BCAS1 expression identifies newly generated oligodendrocytes}

We examined our previously generated proteome database for oligodendrocyte specific proteins and noticed that BCAS1 was one of the most enriched proteins in oligodendrocytes (21). We generated a polyclonal antibody (see Supplementary Materials for details) against BCAS1 and validated its specificity by preabsorption of the antibody with recombinant BCAS1 protein before immunolabeling. Preabsorption eliminated staining of mouse brain sections (fig. S1A). In addition, transfections of Bcas 1 cDNA (complementary DNA) into neuroblastoma cells followed by immunofluorescence staining showed labeling only in transfected cells (fig. S1B), and costaining of the polyclonal antiserum with anti-BCAS1 mouse monoclonal antibody on the mouse and human brain section revealed overlapping staining pattern (fig. S1C). Finally, abolished BCAS1 immunoreactivity on adult Bcas 1 knockout mouse brain sections demonstrated that the staining was specific for BCAS1 (fig. S1D).

Immunofluorescence on frozen mouse tissue sections of the corpus callosum and somatosensory cortex [postnatal day 40 (P40) and 4 months] showed that all $\mathrm{BCAS1}^{+}$cells were of the oligodendrocyte lineage, as shown by colabeling with the lineage markers, sex-determining region Y box 10 protein (SOX10), oligodendrocyte transcription factor 1 (OLIG1), and OLIG2 (Fig. 1, A and B, and fig. S2, A and B). Immunolabeling with antibodies against neural/glial antigen 2 (NG2; a marker for oligodendrocyte progenitor cells) revealed that a small fraction (16.5 \pm $2.8 \%$ ) of the $\mathrm{BCAS1}^{+}$cells were oligodendrocyte progenitor cells (Fig. 1C). The remaining cells were more differentiated, as shown by their reactivity with antibodies against mature oligodendrocytes such as adenomatous polyposis coli protein (76.2 $\pm 3.5 \%$ of overlap) (Fig. 1C; anti-adenomatous polyposis coli antibody, clone CC1) and myelin-associated glycoprotein (MAG; $47.5 \pm 3.2 \%$ of overlap), a protein involved in oligodendrocyte and axon contact (Fig. 1C).

To analyze whether $\mathrm{BCAS1}^{+}$cells were newly formed oligodendrocytes, we performed fate mapping experiments. We crossbred transgenic mice expressing a tamoxifen-inducible Cre DNA recombinase inserted into the NG2 locus [NG2-CreERT2 (22)] with a widely used reporter mouse strain containing a membrane-targeted enhanced green fluorescent protein (EGFP) gene inserted into the Gt(ROSA)26Sor locus (23). NG2-CreERT2 $\times$ R26R-tdTomato-membrane EGFP (mEGFP) mice were injected with tamoxifen at P30 to induce Cre-dependent GFP expression in oligodendrocyte progenitor cells. When we analyzed the progenies of the labeled cells at 7, 18, and 40 days after injection by immunostaining, we observed an increase in the number of cells co-

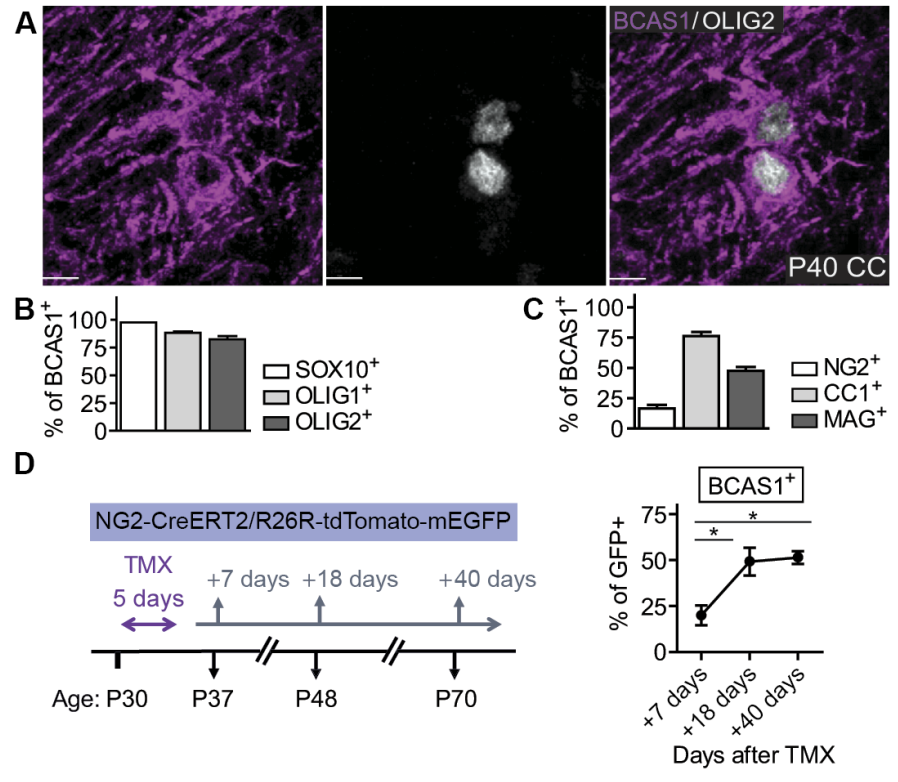

Fig. 1. BCAS1 expression identifies newly generated oligodendrocytes. (A) Confocal images of breast carcinoma amplified sequence 1 (BCAS1) (pink) and oligodendrocyte transcription factor 2 (OLIG2) (white) in the corpus callosum (CC) of postnatal day 40 (P40) mice. Scale bars, $5 \mu \mathrm{m}$. (B) Bar graph shows the percentage of $\mathrm{BCAS}^{+}$cells expressing sex-determining region $\mathrm{Y}$ box 10 protein (SOX10), OLIG1, or OLIG2. (C) Bar graph shows the percentage of $\mathrm{BCAS} 1^{+}$cells expressing neural/glial antigen 2 (NG2), CC1, or myelin-associated glycoprotein (MAG) in the corpus callosum of P40 mice. (D) Left (experimental plan): NG2-CreERT2 $\times$ R26RtdTomato-mEGFP mice were used to induce the expression of mEGFP by tamoxifen $(T M X)$ at P30 as illustrated. Right: Quantification of the percentage of double-positive $\left[\mathrm{GFP}^{+}\right.$(green fluorescent protein-positive) and $\left.\mathrm{BCAS1}^{+}\right]$cells over time. One-way analysis of variance (ANOVA), followed by Bonferroni's post hoc test, ${ }^{*} P<0.05 ; n=3$, three sections per animal. Quantitative data are means \pm SEM.

expressing BCAS1 and GFP from days 7 to 18 in the somatosensory cortex, indicating that $\mathrm{BCAS}^{+}$cells differentiate from oligodendrocyte progenitor cells (Fig. 1D).

\section{$\mathrm{BCAS1}^{+}$premyelinating and myelinating oligodendrocytes are present during development and in adult mice}

Immunofluorescence on somatosensory cortex during postnatal development (P40) showed that BCAS1 was expressed in $\mathrm{CC}^{+}$oligodendrocytes with different morphologies-cells with multiple, symmetrically radiating processes and with several segments aligned in parallel (fig. S3A). Oligodendrocytes that radially extend processes are reminiscent of previously described premyelinating oligodendrocytes at an early stage of the oligodendrocyte differentiation $(24,25)$. To determine whether $\mathrm{BCASl}^{+}$cells with radiating processes represent premyelinating oligodendrocytes, we used transgenic mice, in which the proteolipid protein (PLP) promoter drives the expression of GFP (PLP-GFP) (26). PLP is one of the major structural proteins in myelin and therefore not only highly expressed in mature myelin-forming oligodendrocytes but also present in premyelinating cells during development (24). We detected $\mathrm{GFP}^{+}$premyelinating oligodendrocytes in early postnatal development (P9 and P14), when many oligodendrocytes are formed (Fig. 2, A and B). Whereas all of the $\mathrm{GFP}^{+}$premyelinating oligodendrocytes also showed immunoreactivity against BCAS1, most of the $\mathrm{BCAS}^{+}$premyelinating cells $(70.5 \pm 8.1 \%$ at P9 and $76.3 \pm 1.7 \%$ at P14) did not express GFP, demonstrating that BCAS1 is expressed in a larger population of newly 

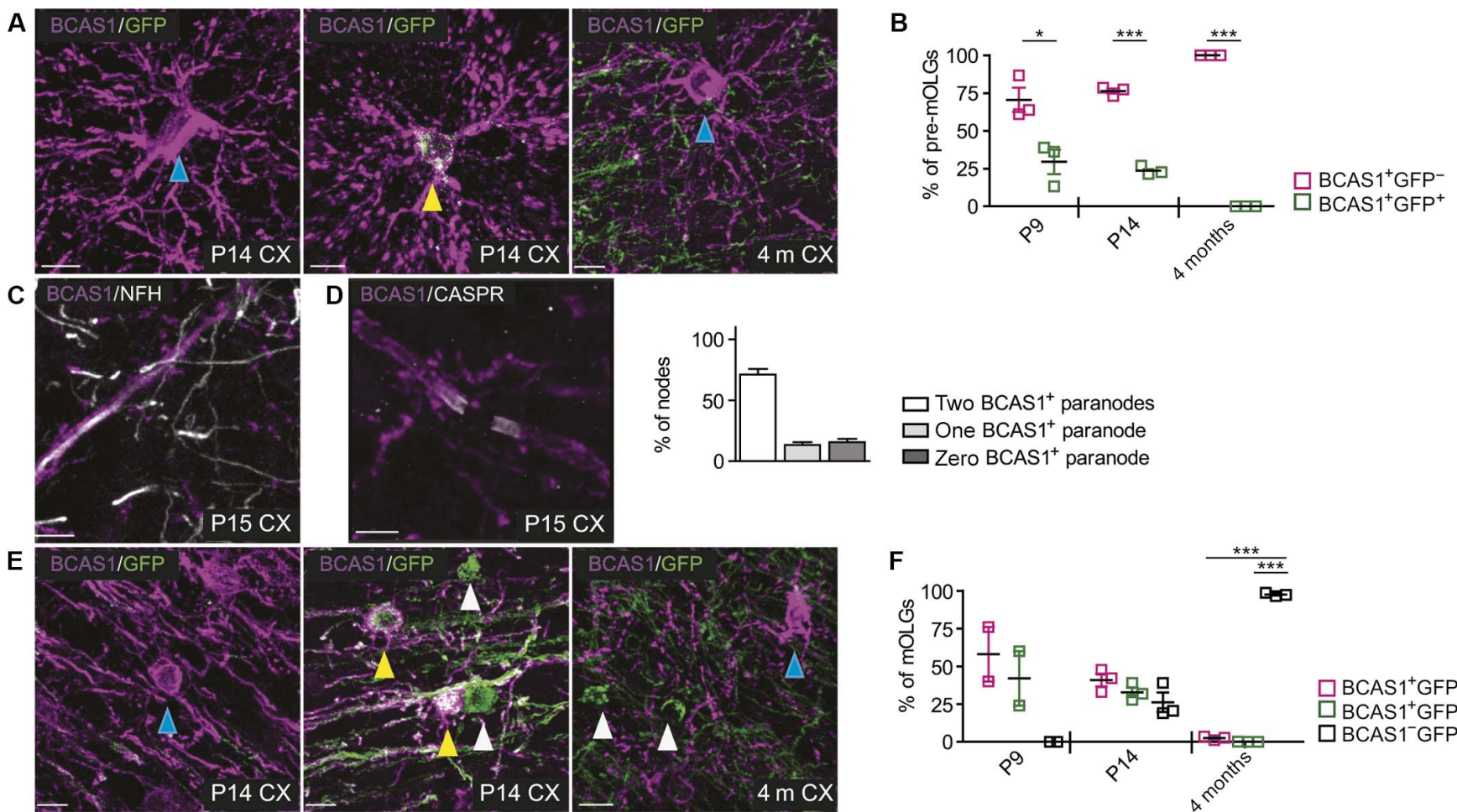

$\mathrm{BCAS1}^{+} \mathrm{GFP}^{-}$ $\square \mathrm{BCAS1}^{+} \mathrm{GFP}^{+}$ $\square \mathrm{BCAS}^{-} \mathrm{GFP}^{+}$

G

OPCs

Pre-mOLGs

mOLGs

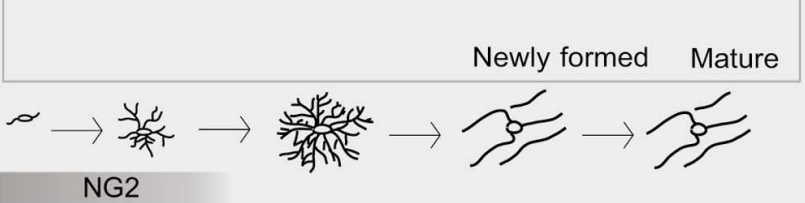

NG2

CC1

BCAS1

H

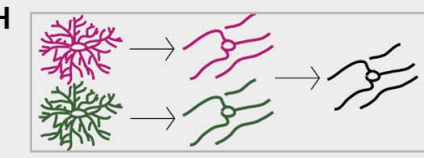

Development

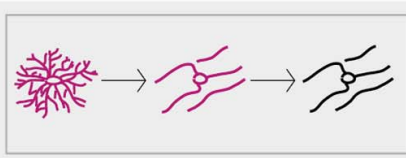

Adulthood

BCAS1 $1^{+}$PLP ${ }^{-}$

BCAS1 $1^{+} \mathrm{PLP} \mathrm{P}^{+}$ Pre-mOLG

(low BCAS1 expression)

Fig. 2. $\mathrm{BCAS1}^{+}$premyelinating and myelinating oligodendrocytes are present during development and in adult mice. (A) Immunohistochemical analysis of transgenic mice in which the proteolipid protein (PLP) promoter drives the expression of GFP (PLP-GFP mice); representative confocal images show BCAS1 (pink) and GFP (green) expression in premyelinating oligodendrocytes (pre-mOLGs) in the developing and adult somatosensory cortex (CX) of P9, P14, and 4-month-old mice. Blue arrows indicate $\mathrm{BCAS1}^{+} / \mathrm{GFP}^{-}$, and yellow arrows indicate $\mathrm{BCAS1}^{+} / \mathrm{GFP}^{+}$pre-mOLGs. Scale bars, $10 \mu \mathrm{m}$. (B) Quantification of the percentage of pre-mOLGs positive for GFP, BCAS1, or both in the somatosensory cortex of P9, P14, and 4-month-old mice. Student's two-tailed $t$ test, unpaired, ${ }^{*} P<0.05$, ${ }^{* * * P}<0.001$. (C) Representative confocal image of $\mathrm{BCAS1}^{+}$segments (pink) aligned along neurofilament-positive (white) axons in the somatosensory cortex of P15 mice. Scale bar, $4 \mu \mathrm{m}$. (D) Left: Representative confocal image of $\mathrm{BCAS1}^{+}$segments (pink) flanked by contactin-associated protein 1 (CASPR) clusters (white) on both sides of a node in the somatosensory cortex of P15 mice. Scale bar, $3 \mu \mathrm{m}$. Right: Quantification of the percentage of nodes in the somatosensory cortex of P15 mice showing BCAS1 ${ }^{+}$segments and CASPR expression on one side (one $\mathrm{BCAS1}^{+}$paranode), both sides (two BCAS1 ${ }^{+}$paranodes), or without BCAS1 ${ }^{+}$segments (zero BCAS1 ${ }^{+}$paranode). (E) Representative confocal images showing BCAS1 (pink) and GFP (green; PLP-GFP mice) expression in myelinating (mOLGs) in the developing and adult somatosensory cortex of P9, P14, and 4-month-old mice. Blue, yellow, and white arrows show BCAS1 ${ }^{+} / \mathrm{GFP}^{-}, \mathrm{BCAS1}^{+} / \mathrm{GFP}^{+}$, and BCAS1 ${ }^{-} / \mathrm{GFP}^{+} \mathrm{mOLGs}$, respectively. Scale bars, $10 \mu \mathrm{m}$. (F) Quantification of the percentage of mOLG positive for GFP, BCAS1, or both in the somatosensory cortex of P9, P14, and 4-month-old mice. Quantitative data are means \pm SEM; $n=3$, three sections per animal [only $n=2$ for P9 in (F)]; one-way ANOVA, followed by Bonferroni's post hoc test, ${ }^{* * *} P<0.001$. (G) Schematic illustration of the phases of OLG maturation and the relative expression of NG2, CC1, and BCAS1. OPCs, oligodendrocyte progenitor cells. (H) Schematic illustration representing the pattern of the expression of BCAS1 and PLP (green) during development and in adulthood.

formed oligodendrocytes in the somatosensory cortex at both P9 and P14 (Fig. 2B). When the analysis was performed in the adult somatosensory cortex (at 4 months), we found that all $\mathrm{BCAS1}^{+}$premyelinating oligodendrocytes were negative for GFP, suggesting that the previously identified $\mathrm{PLP}^{+}$premyelinating oligodendrocytes constitute a subpopulation ( $29.5 \pm 8.1 \%$ at $\mathrm{P9}, 23.7 \pm 1.7 \%$ at $\mathrm{P} 14$, and $0 \%$ at 4 months) of oligodendrocytes present only during development (Fig. 2B). Thus, a $\mathrm{PLP}^{-} / \mathrm{BCAS1}^{+}$premyelinating subpopulation may be involved in continued oligodendrogenesis in the adult.
Next, we analyzed the other major population of $\mathrm{BCAS1}^{+}$oligodendrocytes, the cells with $\mathrm{T}$-shaped processes that resemble myelinating oligodendrocytes by morphology. Because many of these $\mathrm{BCAS1}^{+}$cells contained numerous processes aligned in parallel reminiscent of myelin sheaths, we tested whether these cells were producing myelin segments enwrapping axons. High-resolution confocal images of somatosensory cortex sections confirmed ensheathment of axons by $\mathrm{BCAS1}^{+}$segments as shown by costaining with antibodies against a neurofilament (Fig. 2C). A total of $17.1 \pm 1.9 \%$ of these $\mathrm{BCAS1}^{+}$cells displayed myelin segments 
with a patchy arrangement along the axons resembling the previously reported "intermittent myelin" of cortical pyramidal neurons (fig. S3B) (27). Because the formation of myelin is accompanied by the generation of axon-glial junctions, so-called paranodes, at the edges of the sheaths (28), we immunostained the somatosensory cortex of P15 mice for the neuronal and paranodal protein contactin-associated protein $1 . \mathrm{BCAS1}^{+}$ segments were flanked by contactin-associated protein 1 clusters on one side (one paranode, $17.1 \pm 1.9 \%$ ) or both sides (two paranodes, $62.2 \pm 2.9 \%$ ), indicating the formation of myelin sheaths (Fig. 2D). The ensheathment of axons together with the formation of axon-glial paranodal junctions define the BCAS1 ${ }^{+}$cells with segments aligned in parallel as myelin-forming oligodendrocytes.

To further analyze these cells, we used the PLP-GFP reporter mice to visualize myelinating oligodendrocytes and found that, at P9, all $\mathrm{GFP}^{+}$ myelinating oligodendrocytes were also positive for BCAS1 (Fig. 2, E and $\mathrm{F}$ ). However, when the analysis was performed at $\mathrm{P} 14$, a population of $\mathrm{GFP}^{+}$myelinating oligodendrocytes that lacked BCAS1 expression emerged (Fig. 2, E and F), suggesting that BCAS1 expression is lost when oligodendrocytes mature into later stages of oligodendrocyte differentiation (Fig. 2, G and H). At 4 months, we observed a complete segregation of $\mathrm{GFP}^{+}$and $\mathrm{BCAS1}^{+}$myelinating oligodendrocytes (Fig. 2F).

To verify the segregation of $\mathrm{BCAS1}^{+}$and $\mathrm{PLP}^{+}$oligodendrocytes in adult mice, we crossbred transgenic mice expressing a tamoxifen-inducible Cre inserted into the PLP locus (PLP-CreERT2) with GFP reporter mice (R26R-tdTomato-mEGFP). We injected tamoxifen at P110 to induce GFP expression in oligodendrocytes (fig. S3C). When brain sections were analyzed by immunohistochemistry 18 days after injection, no overlap between $\mathrm{BCAS1}^{+}$and $\mathrm{GFP}^{+}$cell populations was seen in adult mice (fig. S3C). In addition, immunostainings of the somatosensory cortex of adult wild-type mice showed that $\mathrm{BCAS1}^{+}$myelinating oligodendrocytes were only positive for MAG and negative for PLP and that only few $(17.7 \pm 4.3 \%)$ expressed myelin basic protein (MBP), supporting our hypothesis that these cells constitute a distinct population of oligodendrocytes (fig. S3, D to G). Thus, $\mathrm{BCAS}^{+}$cells represent a population of oligodendrocytes that are at an intermediate state of differentiation, segregating from both oligodendrocyte progenitor cells and mature oligodendrocytes.

Ectonucleotide pyrophosphatase/phosphodiesterase 6 (ENPP6) has recently been used as a marker to track the formation of newly formed oligodendrocytes (9). When we analyzed the corpus callosum and the fimbria hippocampi, a further prominent white matter tract, we found a significant increase in ENPP6 ${ }^{+}$cell bodies from P0 to P7 in the fimbria $(P=0.0291)$ and a significant decrease until P21 $(P=0.0405)$. In addition, $\mathrm{BCAS1}^{+}$cells were abundant in the fimbria at $\mathrm{P} 7$ and decreased until P21 (fig. S4, A to C). However, higher densities of BCAS1 ${ }^{+}$cells were identified already at $\mathrm{P} 0$ (in the fimbria), and $\mathrm{BCAS}^{+}$cells continued to be more abundant as compared to ENPP6 ${ }^{+}$cells in the corpus callosum and fimbria at P7 and in the corpus callosum at P21. At P7, $\mathrm{BCAS1}^{+}$cells coexpressing MAG were found to be more abundant than $\mathrm{ENPP}^{+} / \mathrm{MAG}^{+}$cells, indicating that myelin protein expression starts earlier in $\mathrm{BCAS}^{+}$cells (fig. S4, D and E).

\section{$\mathrm{BCAS}^{+}$cells represent a transient population of oligodendrocytes}

To determine the expression of BCAS1 during oligodendrocyte differentiation, we used primary cultures of oligodendrocytes. After $\sim 3$ days in culture, oligodendrocytes progenitor cells start to differentiate into oligodendrocytes with multiple, symmetrically radiating processes, which further mature into with flat myelin-like membrane sheets, in which proteins and lipids of myelin, such as MBP, PLP, and galactosylceramide/ sulfatide (recognized by the antibody O4), are localized. BCAS1 was expressed in $85.9 \pm 2.2 \%$ of the population of $\mathrm{O}^{+}$oligodendrocytes with arborized morphology at 3 days in vitro (fig. S5A). However, after 5 days in culture, when oligodendrocytes formed sheets and expressed MBP, we observed a reduction in BCAS1 immunolabeling (fig. S5B). BCAS1 was virtually excluded from the compacted myelin-like membrane sheets but remained at low levels in the cytosol-enriched, noncompacted areas (fig. S5C). This is in contrast to galactosylceramides/sulfatides (recognized by $\mathrm{O} 1$ and $\mathrm{O} 4$ immunolabeling), another oligodendroglial marker, which remain enriched in myelin sheets of differentiated cells in vitro (fig. S6A) and in vivo (fig. S6B). To extend the analysis to human cells, we generated oligodendrocytes from induced pluripotent human stem cells (iPSCs) derived from healthy human adults (29). We found that BCAS1 was expressed in $86.1 \pm 2.3 \%$ of the $\mathrm{O}^{+}$oligodendrocytes with arborized morphology at 24 days in vitro (fig. S7A). To analyze the cells at a later time point of differentiation, we performed immunolabeling against MBP at 32 days in vitro, when cells have formed myelin-like membrane sheets. We found that only $4 \pm 1.2 \%$ of the fully mature $\mathrm{MBP}^{+}$oligodendrocytes were labeled with antibodies against BCAS1 (fig. S7B), demonstrating the enrichment of BCAS1 at an early differentiation stage in both mouse- and human-cultured oligodendrocytes.

To determine whether BCAS1 is also transiently expressed in oligodendrocytes in vivo, we used 5-ethynyl-2'-deoxyuridine (EdU), a thymidine analog, to pulse-label dividing cells followed by a chase to identify the progeny of dividing cells (Fig. 3, A to D). When EdU was administered for 10 days via the drinking water to 4-month-old mice, $65 \pm 2.7 \%$ of the BCAS1 ${ }^{+}$cells incorporated EdU (Fig. 3B). Notably, after 40 days of a "chase" only, $7.7 \pm 1.3 \%$ of the $\mathrm{EdU}^{+} / \mathrm{BCAS1}^{+}$cells were left revealing their transient nature (Fig. 3B). To exclude dilution of the EdU labeling as the underlying reason, we also visualized mature oligodendrocytes by immunolabeling for $\mathrm{CC} 1$ in the corpus callosum. As expected, we found an increase in the number of $\mathrm{EdU}^{+} / \mathrm{CCl}^{+}$in $\mathrm{BCAS1}^{-}$cells at day 18 (but not significant at day 40), indicating their differentiation into a stable population of oligodendrocytes (Fig. 3D). The number of $\mathrm{EdU}^{+} / \mathrm{CC1}^{+} / \mathrm{BCAS}^{+}$cells, however, decreased with time, confirming our conclusion of their transient nature (Fig. 3D). When similar experiments were performed in the somatosensory cortex, we observed that only $12.1 \pm 1.5 \%$ of the $\mathrm{BCAS1}^{+}$cells incorporated $\mathrm{EdU}$ after 10 days of administration ( +0 days; Fig. $3 \mathrm{C}$ ). In contrast to the corpus callosum, the fraction of $\mathrm{EdU}^{+} / \mathrm{BCAS}^{+}$cells increased slightly to $17.9 \pm 1.1 \%$ after 40 days of a chase ( +40 days; Fig. $3 \mathrm{C}$ ). In addition, analysis of premyelinating and myelinating oligodendrocytes individually showed that the number $\mathrm{EdU}^{+} / \mathrm{BCAS}^{+}$remained stable after the chase (fig. S8, A and B). Thus, $\mathrm{BCAS1}^{+}$cells constitute a transient cell population in the corpus callosum but not in the cortex.

To further explore the dynamics of the $\mathrm{BCAS}^{+}{ }^{+}$oligodendrocytes in the somatosensory cortex, we deleted the establishment of sister chromatid cohesion $\mathrm{N}$-acetyltransferase 2 (Esco2) in the oligodendrocyte lineage using the inducible Sox10-CreERT2 $\times \mathrm{Esco}^{\mathrm{fl} / \mathrm{fl}}$ mouse line (referred to as Sox10-Esco ${ }^{\mathrm{fl}}$ and Sox10-Esco $2^{\mathrm{wt}}$ mice for control) to specifically ablate proliferating oligodendrocyte progenitor cells $(30,31)$. Upon tamoxifen administration, Esco2 is depleted, resulting in a detrimental block of the cohesion apparatus and subsequent apoptosis of all cells undergoing mitosis, that is, proliferating oligodendrocyte progenitor cells. To label the recombined cells, we crossed these mice with the CAG-EGFP reporter mouse line to induce the permanent expression of GFP. Recombination was induced by tamoxifen in adult mice ( 8 to 10 weeks of age), and the number of $\mathrm{GFP}^{+} / \mathrm{BCAS}^{+}$oligodendrocytes 


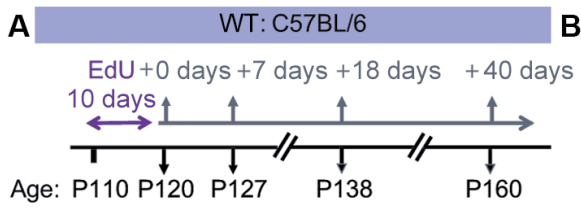

E

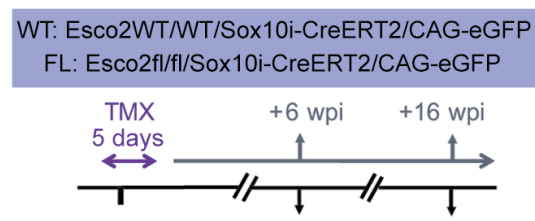

Age: 8 to 10 weeks 14 to 16 weeks 24 to 26 weeks

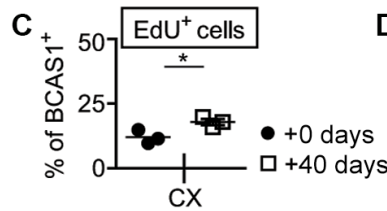

CC $\square+40$ days

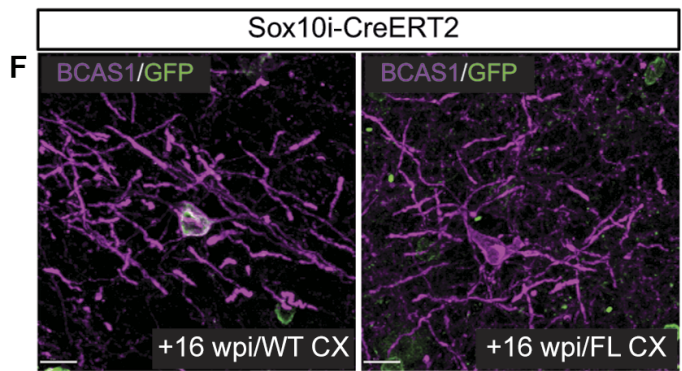

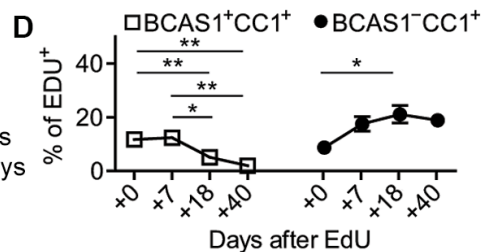

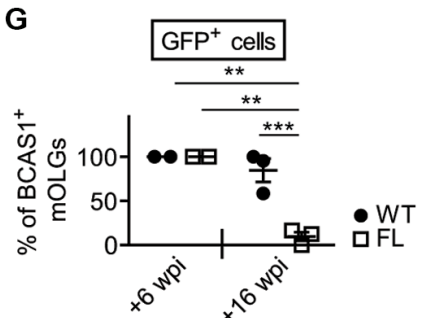

Fig. 3. BCAS1 ${ }^{+}$oligodendrocytes are a transient cell population. (A) Experimental design: 5-Ethynyl-2'-deoxyuridine (EdU) was administered to a 4-month-old mice as shown in the scheme. Analyses were performed 10 (+0 days), 17 (+7 days), 28 (+18 days), and 50 (+40 days) days after injection. (B and C) Quantification of the percentage of $\mathrm{EdU}^{+} / \mathrm{BCAS1}^{+}$cells directly ( 0 days) and 40 days after the end of the treatment in the corpus callosum (B) and somatosensory cortex (C). Student's twotailed $t$ test, unpaired, ${ }^{*} P<0.05,{ }^{* * *} P<0.001$. (D) Line graph showing the time-course expression analysis of the fraction of $C \mathrm{CC} 1^{+}$oligodendrocytes that were EdU ${ }^{+}$and either $\mathrm{BCAS1}^{+}$or $\mathrm{BCAS1}^{-}$plotted versus the time after the end of the EdU exposure in the corpus callosum. One-way ANOVA, followed by Newman-Keuls' post hoc test, ${ }^{*} P<0.05,{ }^{*} P<0.01$. (E) Experimental design: Proliferating OPCs were ablated by tamoxifen injection in inducible Sox 10 -iCreERT2 $\times$ Esco2fl/fl $\times$ CAG-EGFP mice (FL) and compared to control animals Sox10-iCreERT2 $\times$ Esco2wt/wt $\times$ CAG-EGFP (WT). Esco2, establishment of sister chromatid cohesion $\mathrm{N}$-acetyltransferase 2. Tamoxifen was injected in 8- to 10-week-old animals, and analyses were performed 6 and 16 weeks after injection. Recombined cells are marked by EGFP. (F and G) Representative images of BCAS1 and GFP expression in the somatosensory cortex of WT and FL mice 16 weeks after tamoxifen injection (F) and quantification of the percentage of BCAS1 ${ }^{+}$mOLGs that are positive for GFP at 6 and 16 weeks postinduction (wpi) in the somatosensory cortex (G). Scale bars, $15 \mu \mathrm{m}$. Student's two-tailed $t$ test, unpaired, ${ }^{*} P<0.01,{ }^{* *} P<0.001 .6 \mathrm{wpi} / \mathrm{WT}$ versus $16 \mathrm{wpi} / \mathrm{KO}, P=0.0028 ; 6 \mathrm{wpi} / \mathrm{KO}$ versus $16 \mathrm{wpi} / \mathrm{KO}, P=0.0028 ; 16 \mathrm{wpi} / \mathrm{WT}$ versus $16 \mathrm{wpi} / \mathrm{KO}, P=0.0004$. KO, knockout. For all quantifications, $n=3$ (only $n=2$ for the +6 wpi group), three sections per animal. Quantitative data are means \pm SEM.

was quantified (Fig. 3, E to G). Although the number of $\mathrm{GFP}^{+} / \mathrm{BCAS1}^{+}$ myelinating oligodendrocytes cells remained stable at 6 weeks after induction (Fig. 3G), we found a marked reduction in the number of $\mathrm{GFP}^{+} / \mathrm{BCAS1}^{+}$premyelinating oligodendrocytes (reduction from $92.1 \pm$ $3.1 \%$ in control to $29.5 \pm 1.3 \%$ in the knockout; fig. S8C) and $\mathrm{GFP}^{+}$/ $\mathrm{BCAS1}^{+}$myelinating oligodendrocytes (reduction from $97.6 \pm 2.4 \%$ in control to $13.9 \pm 7.3 \%$ in the knockout) when animals were analyzed 16 weeks after induction (Fig. 3, F and G). We therefore conclude that in the cortex, $\mathrm{BCAS1}^{+}$oligodendrocytes also represent a transient cell population being continuously generated from proliferating oligodendrocyte progenitor cells, albeit with a much longer lifetime as compared to the corpus callosum.

\section{Myelination continues into adulthood but declines in aged mice}

To determine the abundance of $\mathrm{BCAS1}^{+}$cells in the white matter (corpus callosum) and gray matter (somatosensory cortex), we compared their density to $\mathrm{NG}^{+}$oligodendrocyte progenitor cells in young (P40) and adult (4 months) animals. BCAS1 staining of the corpus callosum of adult, 4-month-old mice showed a marked reduction of labeled cells compared to younger animals (P40) (fig. S9A). Quantification of the number of $\mathrm{BCAS1}^{+}$cells in the corpus callosum revealed numbers similar to $\mathrm{NG}^{+}$oligodendrocyte progenitor cells at $\mathrm{P} 40$ and at 4 months (fig. S9A). Because of its transient expression, BCAS1 immunoreactivity can serve as a tool to map areas with ongoing oligodendrocyte formation in the brain. Therefore, we determined the number of $\mathrm{BCAS1}^{+}$oligodendrocytes up to old age (21-month-old mice) in the somatosensory and frontal cortex, areas that are known to myelinate relatively late. $\mathrm{BCAS1}^{+}$myelinating oligodendrocytes were found at a density of $715.5 \pm 66.8$ cells $/ \mathrm{mm}^{3}$ in young (P40) mice and remained as an abun- dant cell population in the adult mice (4 and 7 months of age), where they were found in the somatosensory cortex at a density of $379 \pm$ 11.9 cells $/ \mathrm{mm}^{3}$ ( 4 months) and $347.7 \pm 26.6$ cells $/ \mathrm{mm}^{3}$ (7 months; Fig. $4 \mathrm{~A}$ ) and in the frontal cortex at a density of $206.4 \pm 57.8$ cells $/ \mathrm{mm}^{3}$ (7 months; fig. S9B). Only in much older mice (21 months of age) that the number of $\mathrm{BCAS1}^{+}$myelinating oligodendrocytes decreased to low levels in the somatosensory cortex $\left(71.2 \pm 11.7\right.$ cells $/ \mathrm{mm}^{3}$; Fig. $\left.4 \mathrm{~A}\right)$. BCAS1 ${ }^{+}$premyelinating oligodendrocytes were also found at higher densities in the somatosensory cortex of young (P40) and adult (4 and 7 months) mice compared to aged (21 months) mice (Fig. $4 \mathrm{~A})$. These results suggested that generation of myelinating oligodendrocytes continues into adulthood but declines in aged mice.

Using an alternative approach, we used a mass spectrometry method to determine the synthesis rate of myelin proteins from development into old age. This method is based on in vivo stable isotope labeling with amino acids in mice (SILAC) (32). Young (1 month), adult (4 months), and old (18 and 26 months) mice were fed for 30 or 60 days with a diet containing the stable ${ }^{13} \mathrm{C}$ isotope incorporated in lysines $\left({ }^{13} \mathrm{C}\right.$-lysines) (Fig. 4B). Subsequently, myelin was purified from isolated brains by density centrifugation, and the incorporation of ${ }^{13} \mathrm{C}$-lysines into proteins was compared to the amount of native ${ }^{12} \mathrm{C}$-lysines by mass spectrometry to obtain a measure of their relative synthesis rates. The ${ }^{13} \mathrm{C}-1$ ${ }^{12} \mathrm{C}$-lysine ratio of the three most abundant proteins of compacted myelin- MBP, PLP, and claudin11-was compared to the ratio of the three major structural proteins of axons-neurofilament light, medium, and heavy chains-that copurify with myelin. Although the calculation of the ${ }^{13} \mathrm{C} /{ }^{12} \mathrm{C}$-lysine ratios revealed that the metabolism of neurofilaments had reached steady state in 4-month-old animals and remained at similar level throughout the life, we found that this was not the case for myelin proteins. The incorporation of the ${ }^{13} \mathrm{C}$-lysines into myelin 


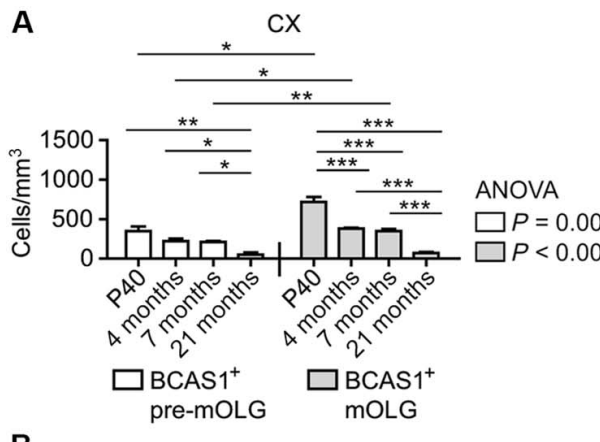

B

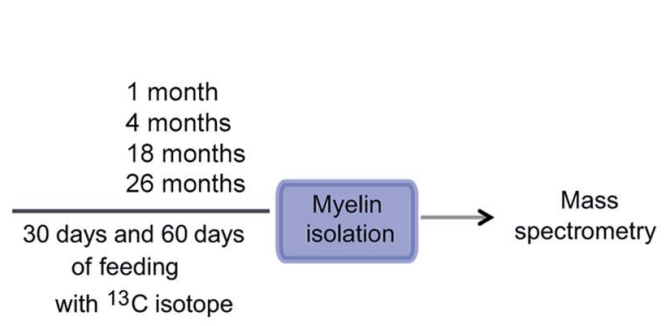

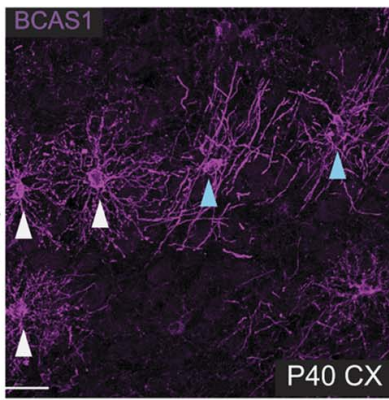
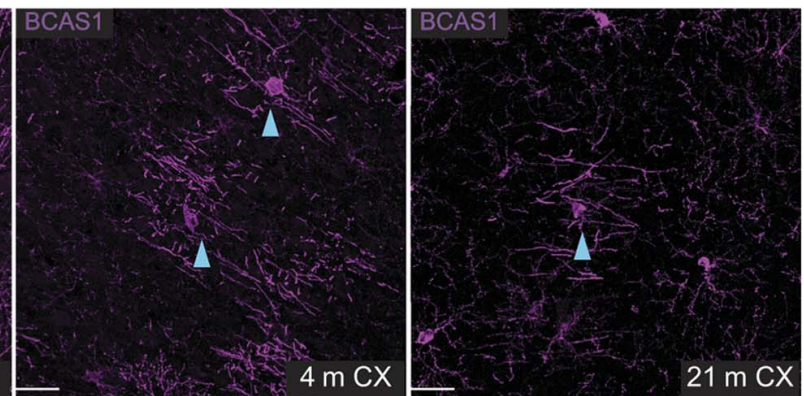
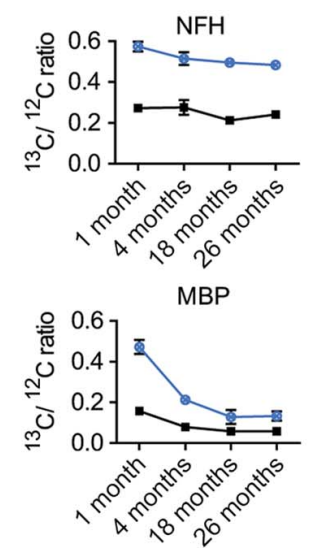

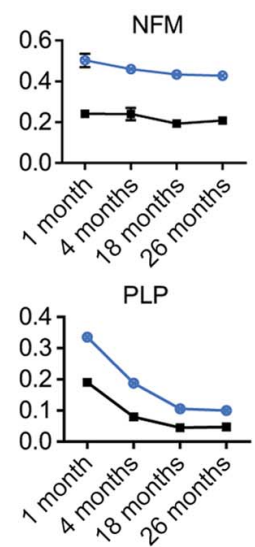

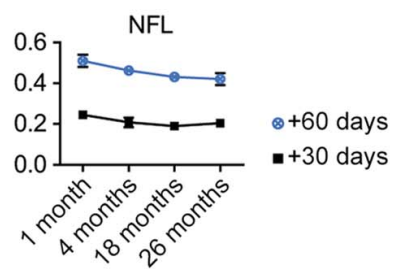

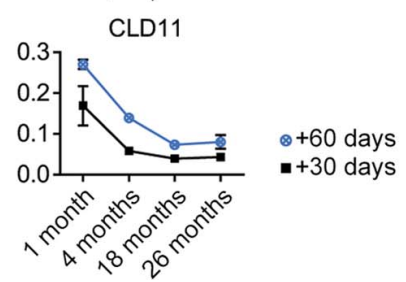

Fig. 4. Myelination continues into adulthood but decreases in aged mice. (A) Cell density quantification (left) and immunohistochemistry (right) of $B C A S 1^{+}$premOLGs and mOLGs in the somatosensory cortex in P40 and 4-, 7-, and 21-month-old mice. Scale bars, $30 \mu \mathrm{m}$. White and blue arrows indicate BCAS1 ${ }^{+}$pre-mOLGs and $B C A S 1^{+}$mOLGs, respectively. One-way ANOVA, followed by Newman-Keuls' post hoc test, ${ }^{*} P<0.05$, ${ }^{* *} P<0.01$. Student's two-tailed $t$ test, unpaired, for intergroup analysis, ${ }^{*} P<0.05,{ }^{* * *} P<0.001 . n=3$, three sections per animal. Quantitative data are means \pm SEM. (B) Left (experimental design): Mass spectrometry analysis was performed in $1-, 4-, 18$-, and 26 -month-old mice fed for 30 or 60 days with a diet containing lysine labeled with ${ }^{13} \mathrm{C}\left({ }^{13} \mathrm{C}\right.$-isotope). Right: The ${ }^{13} \mathrm{C}$ isotope incorporation rates $\left({ }^{13} \mathrm{C}\right.$-lysines/ ${ }^{12} \mathrm{C}$-lysines ratio) are shown for three axonal proteins-neurofilament light (NFL), neurofilament medium (NFM), and neurofilament heavy (NFH) chains-and for the three major myelin proteins-PLP, myelin basic protein (MBP), and CLD11; four animals per group, 4-month-old groups versus 18- and 26-month-old groups, one-way ANOVA, followed by Bonferroni's post hoc test. 30 days feeding: $P<0.0001, \mathrm{PLP} ; P=0.0226, \mathrm{MBP} ; P<0.0001, \mathrm{CLD} 11 ; P=0.0800, \mathrm{NFH} ; P=0.2355, \mathrm{NFM} ; P=0.2700, \mathrm{NFL}$. 60 days feeding: $P<$ 0.0001 , PLP; $P=0.0016, \mathrm{MBP} ; P<0.0001, \mathrm{CLD} 11 ; P=0.1338, \mathrm{NFH} ; P=0.0671, \mathrm{NFM} ; \mathrm{NFL}, P=0.0229 . n=4$, three technical replicates. Error bars indicate SD.

proteins occurred at a higher rate in 1- and 4-month-old mice when compared to 18- and 26-month-old animals (Fig. 4B). Overall, the high ratios of ${ }^{13} \mathrm{C}-{ }^{12} \mathrm{C}$-lysines in myelin proteins are consistent with prominent myelination at 1 month, still active myelination in 4-month-old mice but reduced myelin protein synthesis in aged mice. Thus, our SILAC-based quantification provides further evidence that myelination continues into adulthood but declines in aged mice.

One of the possible reasons for adult myelination could be the extensive turnover of myelinating oligodendrocytes in adult mice. Hence, we used the PLP-CreERT $2 \times$ R26R-tdTomato-mEGFP reporter mice to induce the expression of EGFP by tamoxifen at P30 in oligodendrocytes and to track the fate of developmentally born oligodendrocytes. We followed these animals for up to 12 months of age and found an increase in EGFP signal intensity with time in corpus callosum and somatosensory cortex (fig. S10, A to D). When we quantified the density of $\mathrm{GFP}^{+}$cells in the somatosensory cortex of tamoxifen-treated transgenic animals, we found that the number of $\mathrm{GFP}^{+}$oligodendrocytes labeled 18 days after tamoxifen injection did not change over the 11 months of chase, suggesting that developmentally born myelinating oligodendrocytes survive into late adulthood and expand their myelin sheaths (fig. S10, E and F). Hence, these results do not support an extensive turnover of myelinating oligodendrocytes but rather suggest that the generation of new myelin sheaths in the adult is likely due to a maturation or adaptive process that continues into adulthood in mice.
$\mathrm{BCAS1}^{+}$oligodendrocytes are largely restricted to the fetal and early postnatal human white matter but are numerous in the gray matter until old age

Next, we set out to determine whether the results obtained from rodents also hold true for humans. Although a previous work provided evidence that the number of oligodendrocytes remains relatively stable throughout human life (15), tools to visualize actively myelinating oligodendrocytes in human brain are lacking. When newborn human autopsy cases $(n=3)$ were stained with BCAS1 antibodies, we observed, as in mice, cells with multiple and branched processes (Fig. $5 \mathrm{~A}$ ). $\mathrm{BCAS1}^{+}$cells coexpressed OLIG2 and SOX10, suggesting oligodendroglial origin (Fig. 5, $\mathrm{A}$ and $\mathrm{B})$. On the contrary, only $3.5 \pm 1.4 \%$ of the $\mathrm{BCAS1}^{+}$cells was also positive for NG2 (Fig. 5, A and B), and no cells were positive for the proliferation marker Ki67 (fig. S11A). When we performed colabeling with markers for mature oligodendrocytes, tubulin polymerization promoting protein (TPPP/p25), and CC1, only very limited $(0.6 \pm 0.3 \%$ for TPPP/p25) or no overlap (0\% for CC1), was seen (Fig. 5, A and B). We also colabeled BCAS1 or NG2 with ENPP6. In human tissue, ENPP6 colocalized with NG2 $(74.4 \pm 5.3 \%)$, and the overlap with BCAS1 was limited (12.2 $\pm 3.8 \%$; fig. S11, B to F). We found that $\mathrm{BCAS}^{+}{ }^{+}$cells displayed multiple, symmetrically radiating processes or segments aligned in parallel closely resembling the morphology observed in mice. As in mice, $\mathrm{BCAS1}^{+}$cell bodies were not labeled with antibodies against PLP, whereas some cells were stained with antibodies against MAG $(9.8 \pm 2.4 \%$ 


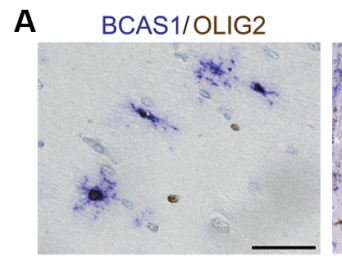

C
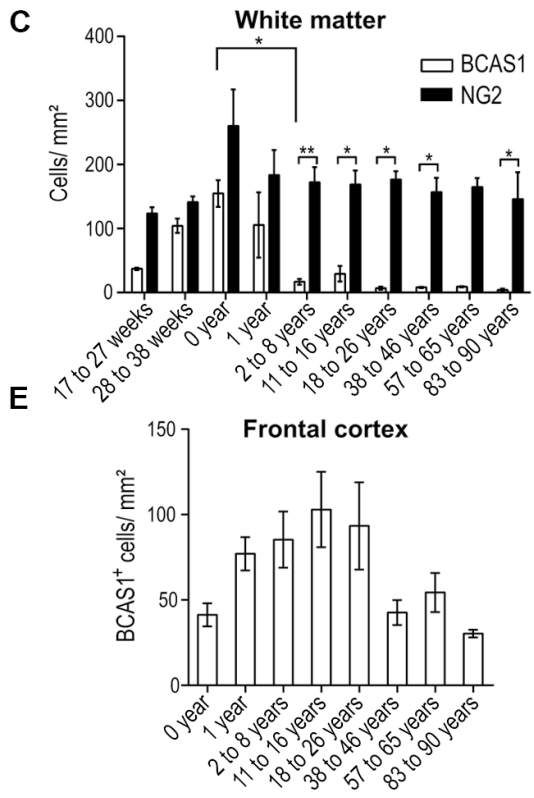

BCAS1/NG2

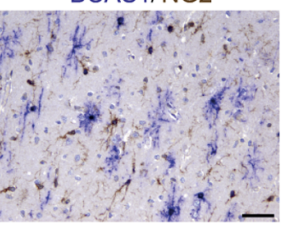

D

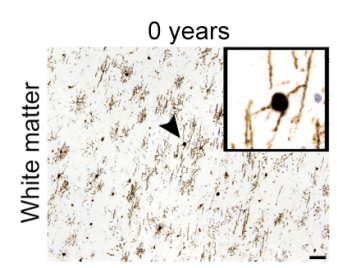

$\mathbf{F}$

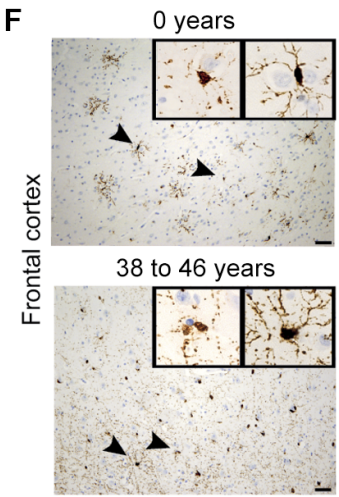

BCAS1/CC1

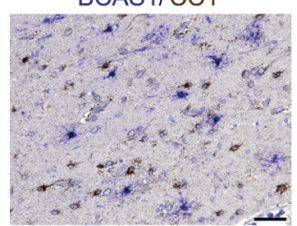

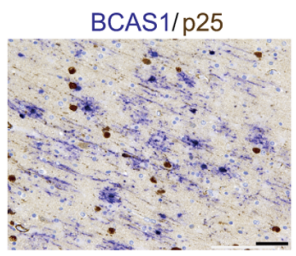
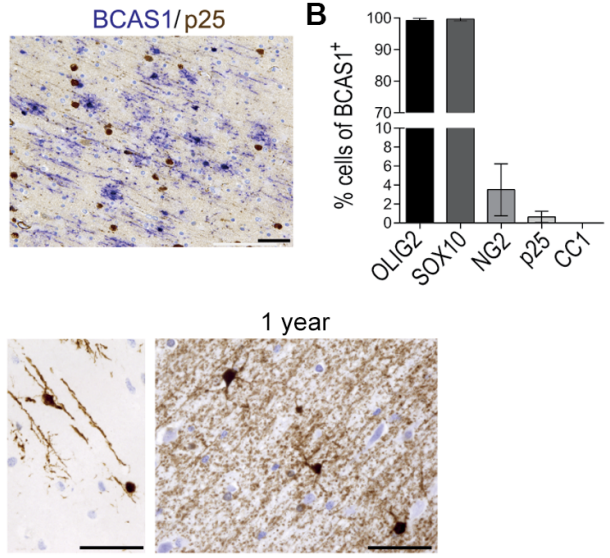

2 to 8 years

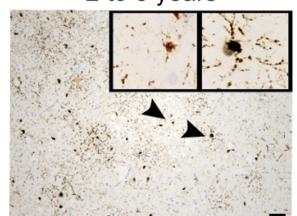

G

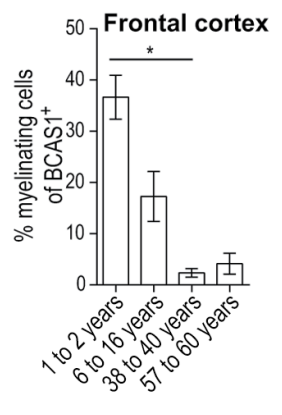

Fig. 5. $\mathrm{BCAS1}{ }^{+}$cells represent a transient oligodendroglial subpopulation enriched during active myelination in the human brain. (A) Immunohistochemistry showing colocalization of BCAS1 with OLIG2, NG2, CC1, and tubulin polymerization promoting protein (TPPP/p25). (B) Quantification of the percentage of BCAS1 ${ }^{+}$cells positive for SOX10, OLIG2, NG2, TPPP/p25, and CC1 in newborns ( $n=3$ to 4). (C) Quantification of the density of BCAS1 ${ }^{+}$and $\mathrm{NG}^{+}$cells in the white matter throughout development and adult life (BCAS1: 17 to 27 weeks, $n=2$; all other time points, $n=3$ to $5 ; \mathrm{NG2}, n=2$ to 5 per time point; Mann-Whitney test, ${ }^{*} P<0.05$, ${ }^{* *} P<0.01 ; \mathrm{BCAS1}$ 0 year versus BCAS1 2 to 8 years, $P=0.0357 ; B C A S 12$ to 8 years versus NG2 2 to 8 years, $P=0.0079 ; B C A S 111$ to 16 years versus NG2 11 to 16 years, $P=0.0159 ; B C A S 1$ 18 to 26 years versus NG2 18 to 26 years, $P=0.0179$; BCAS1 38 to 46 years versus NG2 38 to 46 years, $P=0.0357 ; B C A S 183$ to 90 years versus NG2 83 to 90 years, $P=$ 0.0286). (D) BCAS1 expression in the white matter around birth (0 year) and at 1 year of age (1 year). Inset and image in the center show magnification of the BCAS1 ${ }^{+}$ cells in a newborn human, as indicated by the arrowhead. (E) Quantification of BCAS1 ${ }^{+}$cell density in the frontal cortex $(n=3$ to 6$)$. (F) Representative images of BCAS1 expression in the frontal cortex in humans around birth ( 0 year) and at 2 to 8,38 to 46 , and 57 to 65 years of age (as indicated). The insets show magnifications of BCAS1 ${ }^{+}$cells, as indicated by arrowheads, with few or no segments (left) and with several segments (right). (G) Percentage of BCAS1 ${ }^{+}$cells with a myelinating morphology in the frontal cortex in humans at different ages; $n=3$ to 5 . Kruskal-Wallis test with Dunn's post hoc test, ${ }^{*} P<0.05$. Quantitative data are means \pm SEM. Scale bars, $50 \mu \mathrm{m}$.

of overlap) and MBP ( $7.5 \pm 1.4 \%$ of overlap) (fig. S11, G to I). Hence, we found $\mathrm{BCAS}^{+}$oligodendrocytes to be distinct from $\mathrm{NG}^{+}$oligodendrocyte progenitor cells, $\mathrm{PLP}^{+}$premyelinating oligodendrocytes, and mature oligodendrocytes. We therefore concluded that BCAS1 immunoreactivity identifies a unique stage of oligodendrocyte differentiation in the human brain. We mapped the distribution and the density of $\mathrm{BCAS1}^{+}$cells in the gray and white matter throughout life in human brains (Fig. 5, C to G, fig. S12, and table S1). BCAS1 ${ }^{+}$cells started to appear in the white matter at a gestational age of 17 to 27 weeks $\left(37 \pm 1.7 \mathrm{cells} / \mathrm{mm}^{2}\right)$ and reached their highest values in newborns $\left(154.7 \pm 20.8 \mathrm{cells} / \mathrm{mm}^{2}\right)$ before dropping to $16.8 \pm 4.5$ cells $/ \mathrm{mm}^{2}$ in 2- to 8 -year-old children. Their density reached $6.8 \pm 2.3$ cells $/ \mathrm{mm}^{2}$ in 18 - to 26 -year-old individuals and remained low until old age ( 83 to 90 years old) (Fig. 5C). At 1 year of age, abundant $\mathrm{BCAS1}^{+}$myelin sheaths were observed in the white and gray matter (Fig. 5D and fig. S12), further suggesting that BCAS1 ${ }^{+}$ cells are likely to represent myelinating oligodendrocytes. In addition, the number of $\mathrm{BCAS1}^{+}$cells correlated with the established typical chronological sequence of myelination in the white matter, suggesting that these cells might be useful for identifying active myelination. As a comparison, we quantified the amount of $\mathrm{NG}^{+}$oligodendrocyte progenitor cells in the white matter and found that the number of $\mathrm{NG}^{+}$cells also peaked in newborns $\left(260 \pm 57.3 \mathrm{cells} / \mathrm{mm}^{2}\right)$, but in contrast to BCAS1 ${ }^{+}$cells, the density of $\mathrm{NG2}^{+}$oligodendrocyte progenitor cells remained stable (166 \pm 24 cells $/ \mathrm{mm}^{2}$ ) throughout life into old age (Fig. 5C and fig. S12). Our results suggest that, in contrast to $\mathrm{NG}^{+}$oligodendrocyte progenitor cells, $\mathrm{BCAS1}^{+}$cells are a transient cell population preferentially expressed during the active phase of myelination in the human white matter.

Brain regions controlling more complex tasks, such as the frontal cortex, generate myelin relatively late. We therefore determined the number of $\mathrm{BCAS1}^{+}$oligodendrocytes in the human frontal cortex and found the highest densities during developmental myelination in adolescence and young adulthood (Fig. 5, E and F). However, in contrast to the white matter, the cell density did not change substantially, ranging from 30 to 90 cells $/ \mathrm{mm}^{2}$ throughout adult life (Fig. 5, E and F). We determined the fraction of cells with a myelinating morphology (segments aligned in parallel) and found the highest density in the 1- to 2-year-old individuals ( $36.7 \pm 4.3 \%$ of all BCAS1 ${ }^{+}$cells), followed by the 6 - to 16 -year-old individuals $(17.3 \pm 4.9 \%)$, and 2 to $4 \%$ in the 38 - to 40 -year-old 
and 57- to 60-year-old individuals (Fig. 5G). These results contrast with the white matter tracts and point to the possibility of adult adaptive myelination in the human frontal cortex.

\section{$\mathrm{BCAS1}^{+}$oligodendrocytes are reformed in a proportion of chronic white matter lesions in MS}

Having shown that $\mathrm{BCAS}^{+}$oligodendrocytes are enriched in the fetal and early postnatal white matter, we asked whether they are reformed after a demyelinating event. We first used a mouse model of focal demyelination in the white matter induced by lysolecithin (lysophosphatidylcholine) injection. In lesioned animals, demyelination is complete within 4 days, followed by a repair process that is maximal between 2 and 3 weeks after injection. We found that $\mathrm{BCAS1}^{+}$cell density increased from days 3 to 12 after injection in the phase of most intense remyelination (Fig. 6, A and B). We repeated the analysis in a chronic model of demyelination, in which mice are fed with a demyelinating toxin, cuprizone, for 6 weeks. We observed that $\mathrm{BCAS1}^{+}$cells decreased in the first week of feeding but already started to increase at week 3 and reached their highest densities at week 4 , coinciding with remyelination (Fig. 6, C and $\mathrm{D}$ ).

Next, we asked whether $\mathrm{BCAS1}^{+}$oligodendrocytes are also reformed in human MS lesions. In MS, damaged myelin can be replaced by new sheaths, but the ability to do so is limited (8). Thus far, weaker myelin staining, so-called shadow plaques, is taken as evidence for remyelination, but this analysis does not provide information on the time point of remyelination $(33,34)$. We found that completely demyelinated chronic lesions in the white matter only rarely contained $\mathrm{BCAS}^{+}$oligodendrocytes (Fig. 7A and fig. S13A), consistent with the notion that remyelination is incomplete in MS (16). In contrast, partially remyelinated lesions, as shown by the pale myelin staining at the borders of the lesions, contained clusters of $\mathrm{BCAS1}^{+}$oligodendrocytes (Fig. 7, A to E). The density
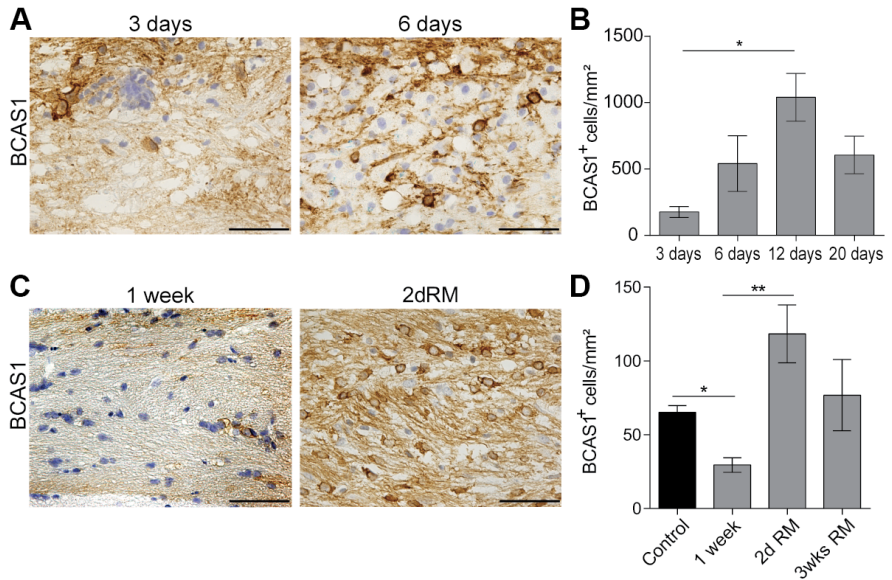

Fig. 6. $\mathrm{BCAS1}^{+}$oligodendrocytes are reformed during remyelination in rodents. (A) Representative images of BCAS1 expression of lysolecithin-induced lesions in the rat corpus callosum on days 3 and 6 after injection. BCAS1 ${ }^{+}$cells are shown in brown, and cell nuclei are shown in blue. (B) Quantification of BCAS1 ${ }^{+}$ cell density in lysolecithin-induced lesions 3, 6, 12, and 20 days after injection ( $n=3$; Kruskal-Wallis test with Dunn's post hoc test, ${ }^{*} P<0.05$ ). (C) Representative images of BCAS1 expression in the corpus callosum 1 and 4 weeks after the start of cuprizone treatment in mice. BCAS1 ${ }^{+}$cells are shown in brown, and cell nuclei are shown in blue. (D) Quantification of BCAS1 ${ }^{+}$cell density in the corpus callosum 1 week after the start of cuprizone treatment and 2 days remyelination ( $2 \mathrm{~d}$ RM) and 3 weeks remyelination (3wks RM) after the treatment was discontinued; $n=4$ to 14 animals. Kruskal-Wallis test with Dunn's post hoc test, ${ }^{*} P<0.05,{ }^{* *} P<0.01$. Quantitative data are means \pm SEM. Scale bars, $50 \mu \mathrm{m}$. of the $\mathrm{BCAS1}^{+}$oligodendrocytes was highest at the lesion border and within the remyelinating areas but low in the lesion core, suggesting that remyelination starts at the lesion border in the white matter (Fig. 7, A to $\mathrm{H})$. $\mathrm{BCAS}^{+}$oligodendrocytes were also increased in the normalappearing white matter surrounding chronic lesions with remyelination (fig. S13). Our analysis of MS lesions from patients up to 74 years of age showed that the capacity to generate or maintain $\mathrm{BCAS}^{+}$oligodendrocytes is retained until old age (table S2). Finally, we examined lesions that were fully remyelinated (completely covered with pale myelin corresponding to shadow plaques) and found that such lesions contained low densities of BCAS1 ${ }^{+}$oligodendrocytes (Fig. 7, A to I, and fig. S13).

Thus, $\mathrm{BCAS}^{+}$oligodendrocytes are found in the predicted chronological sequence of remyelination in human MS lesions, suggesting that a proportion of these cells are engaged in actively reforming myelin. Finally, we determined whether inflammatory activity was a prerequisite for the presence of $\mathrm{BCAS1}^{+}$oligodendrocytes in chronic MS lesions. MS lesions were classified on the basis of the presence or absence of macrophages/activated microglia (inflammatory activity) and the presence or absence of ongoing demyelination (demyelinating activity) (Fig. 8, A and $\mathrm{B}$ ). We found that $\mathrm{BCAS}^{+}$oligodendrocytes were not only found in lesions with ongoing inflammatory and/or demyelinating activity but also in completely inactive lesions (Fig. 8, C and D), suggesting that recruitment of oligodendrocyte progenitor cells and stimulation of remyelination are at least in part independent of lesion activity.

\section{DISCUSSION}

The systematic classification of all neural cell types is a central goal toward deconstructing the CNS into its basic components. Del Rio Hortega grouped oligodendrocytes into different subtypes on the basis of their morphological characteristics such as the number and orientation of their cellular processes. Recent single RNA sequencing transcriptome analyses confirm oligodendrocyte heterogeneity $(35,36)$. Nonetheless, molecular tools to distinguish the cellular subtypes or stages of the oligodendrocyte lineage are lacking. Currently, we are largely restricted to the discrimination of oligodendrocyte progenitor cells from mature oligodendrocytes in the human brain (37-39). ENPP6 has recently been used as a marker to track the formation of newly formed oligodendrocytes in mice (9); however, it may not be suitable for this purpose in human tissue sections, as demonstrated in the present work. $\mathrm{PLP}^{+}$premyelinating oligodendrocytes were detected in the developing and diseased human brain, but they seem to represent a minor cell population of newly formed oligodendrocytes $(24,25)$. In addition, most markers, such as $\mathrm{O} 1$ or $\mathrm{O} 4$, do not detect newly formed oligodendrocytes in the adult and are more appropriate to the analysis of the developing brain (40). Thus, to measure myelination and remyelination in human adults, we have thus far been limited to indirect methods, such as magnetic resonance imaging in vivo and electron microscopy or semiquantitative histochemical staining techniques in the tissue. In addition, as of yet, no marker for actively ongoing remyelination exists, and consequently, it has thus far not been possible to determine when new myelin sheaths are reformed in MS (33). Here, we identified $\mathrm{BCAS}^{+}$oligodendrocytes in the mouse and human brain that represent a population of newly generated and early oligodendrocytes transiently present during the active phase of myelination and might thus be used to report areas of active myelination and remyelination. BCAS1 is a relatively short-lived protein with a lifetime of $\sim 18$ days [ 155 days for MBP and 188 days for PLP (calculations from our in vivo SILAC experiments); see Materials and Methods] that resides in 
A
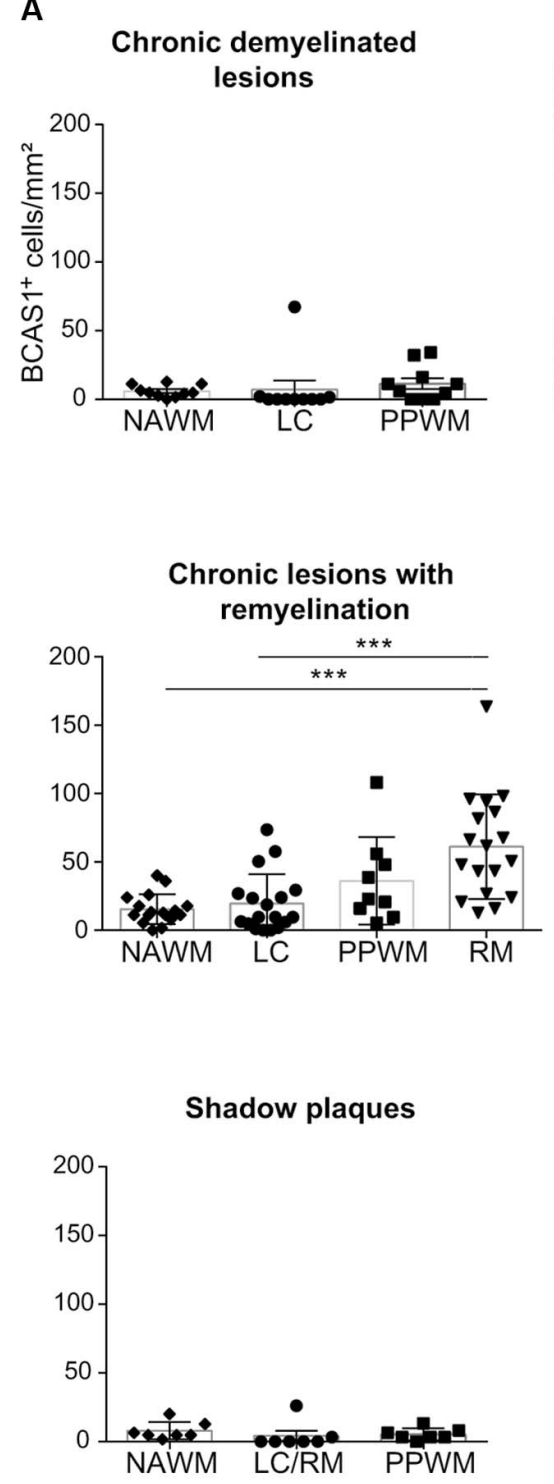
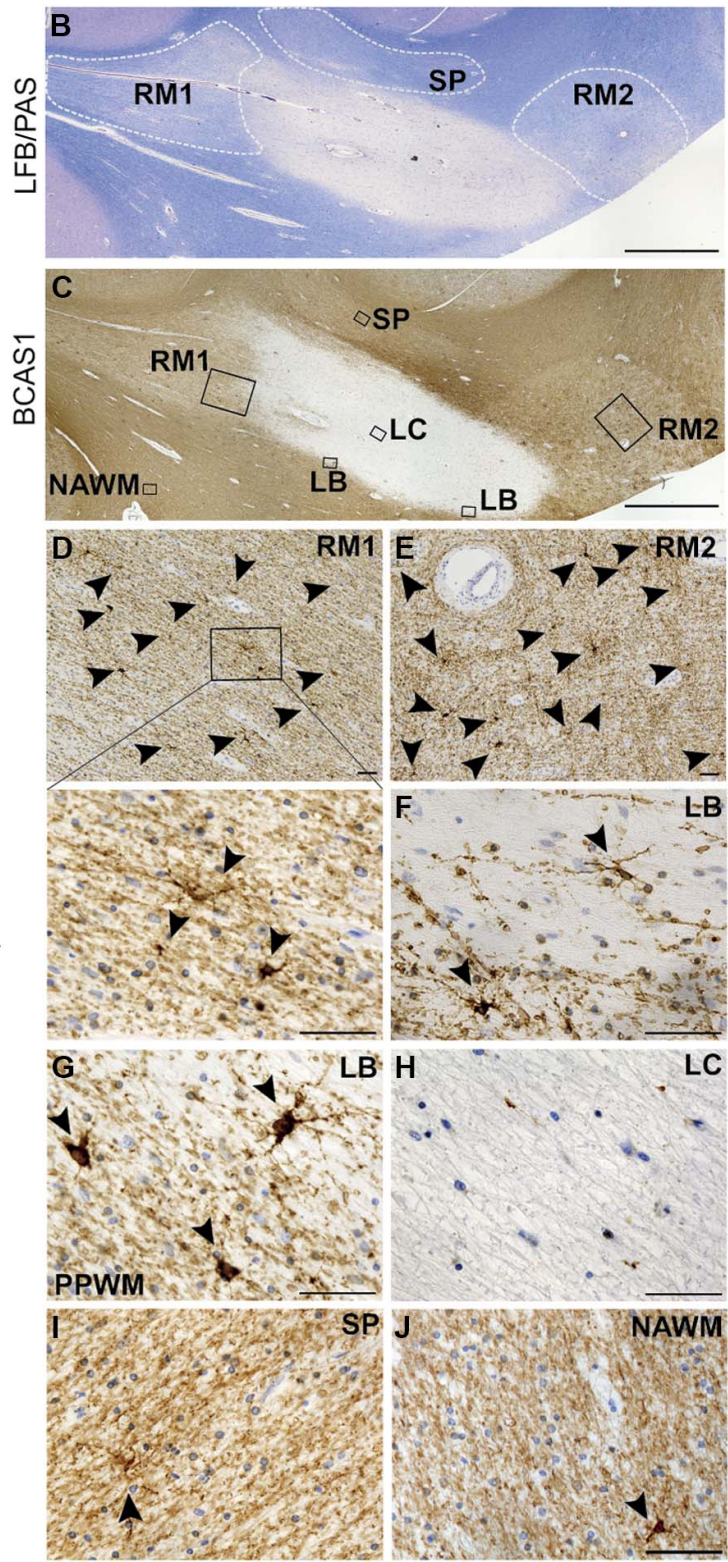

Fig. 7. $B C A S 1^{+}$oligodendrocytes identify areas of ongoing remyelination in chronic multiple sclerosis. (A) Quantification of $\mathrm{BCAS}^{+}$cell density in the normal-appearing white matter (NAWM), the immediate periplaque white matter (PPWM), the lesion center (LC), and remyelinated lesion areas (RM), if present, of chronic demyelinated lesions ( $n=10)$, chronic lesions with areas of remyelination at the lesion edge $\left(n=18\right.$; $\left.{ }^{* *} P<0.001\right)$, and fully remyelinated shadow plaques $(n=7)$. Kruskal-Wallis test with Dunn's post hoc test was used for statistical analysis. (B) Luxol fast blue/periodic acid-Schiff (LFB/PAS) histochemistry depicts the frontal subcortical white matter containing a chronic demyelinated multiple sclerosis lesion with two adjoining sharply delineated lesion areas of pale LFB staining (RM1 and RM2) and a neighboring lesion area with more intense but still visibly reduced LFB staining [shadow plaque (SP)]. (C) BCAS1 immunohistochemistry on a serial section of (B). Frames in (C) and (D) indicate lesion areas depicted at a higher magnification below. LB, lesion border. (D to $\mathbf{J}$ ) Multiple sclerosis lesion areas as indicated in (C) immunostained for BCAS1. Arrowheads indicate $\mathrm{BCAS1}^{+}$cells. Error bars indicate SEM. Scale bars, $2 \mathrm{~mm}$ (B and C) and $50 \mu \mathrm{m}$ (all other images).

the cytosolic spaces of oligodendrocytes and myelin and is downregulated with differentiation. BCAS1 immunoreactivity is first seen in the soma but later appears in newly formed myelin sheaths, suggesting that $\mathrm{BCAS}^{+}$cells might represent myelinating oligodendrocytes. Whereas a fraction of the $\mathrm{BCAS}^{+}$cells represent oligodendrocyte progenitor cells in mice, as determined by NG2 colabeling, the overlap is less than $5 \%$ in humans. In addition, $\mathrm{BCAS}^{+}$oligodendrocytes are also distinct from TPPP $/ \mathrm{p}^{2} 5^{+}$and $\mathrm{CC}^{+}$mature human oligodendrocytes but overlap fully with SOX10 and OLIG2, demonstrating that $\mathrm{BCAS1}^{+}$cells are segregated from other known oligodendroglial subpopulations. In mice but not in humans, a large fraction of the $\mathrm{BCAS1}^{+}$cells colocalized with $\mathrm{CC} 1$; this difference is possibly attributed to differences in the expression pattern of CC1 in mice and human. Quantifying the density of $\mathrm{BCAS1}^{+}$cells, we found a peak in the first year of postnatal white matter development, although they continued to be present in relatively large quantities in the frontal human cortex into old age. These data are in good agreement with the birth dating of oligodendrocytes by ${ }^{14} \mathrm{C}$ integration, which showed that the number of oligodendrocytes in the white matter is largely established during the first years, whereas gray matter oligodendrocytes continue to be generated in adulthood (15). The number of BCAS1 ${ }^{+}$cells also declines in the white matter of adult mice, but in contrast to humans, they were present in relatively high density into adulthood before dropping to very low levels in old age. Differences between mouse and human oligodendrocytes are likely to exist; however, currently, there are no tools available to determine whether myelinating oligodendrocytes are formed from oligodendrocyte progenitor cells or preexisting immature oligodendrocytes in humans. Thus, we cannot exclude the interesting possibility that $\mathrm{BCAS}^{+}$gray matter oligodendrocytes are long-lived and represent a precursor cell population for (re)myelination in the human brain.

In the prototypic human inflammatory demyelinating disease MS, remyelination is considered the prime neuroprotective treatment strategy (8). Our work suggests that active remyelination at the lesion edge is ongoing in a proportion of established, fully demyelinated MS lesions, even at advanced stages of the disease. Thus, older patients are likely to remain receptive to regenerative therapies, which is in line with the conclusions drawn from experimental mouse models (41). Surprisingly, we found that $\mathrm{BCAS1}^{+}$oligodendrocytes were not only found in lesions with ongoing inflammatory and/or demyelinating activity but also in completely inactive lesions.

There are a number of limitations to the present study. Oligodendroglial morphology, metabolism, and function are more complex and less well understood in humans as compared to rodents (42). In addition, the 


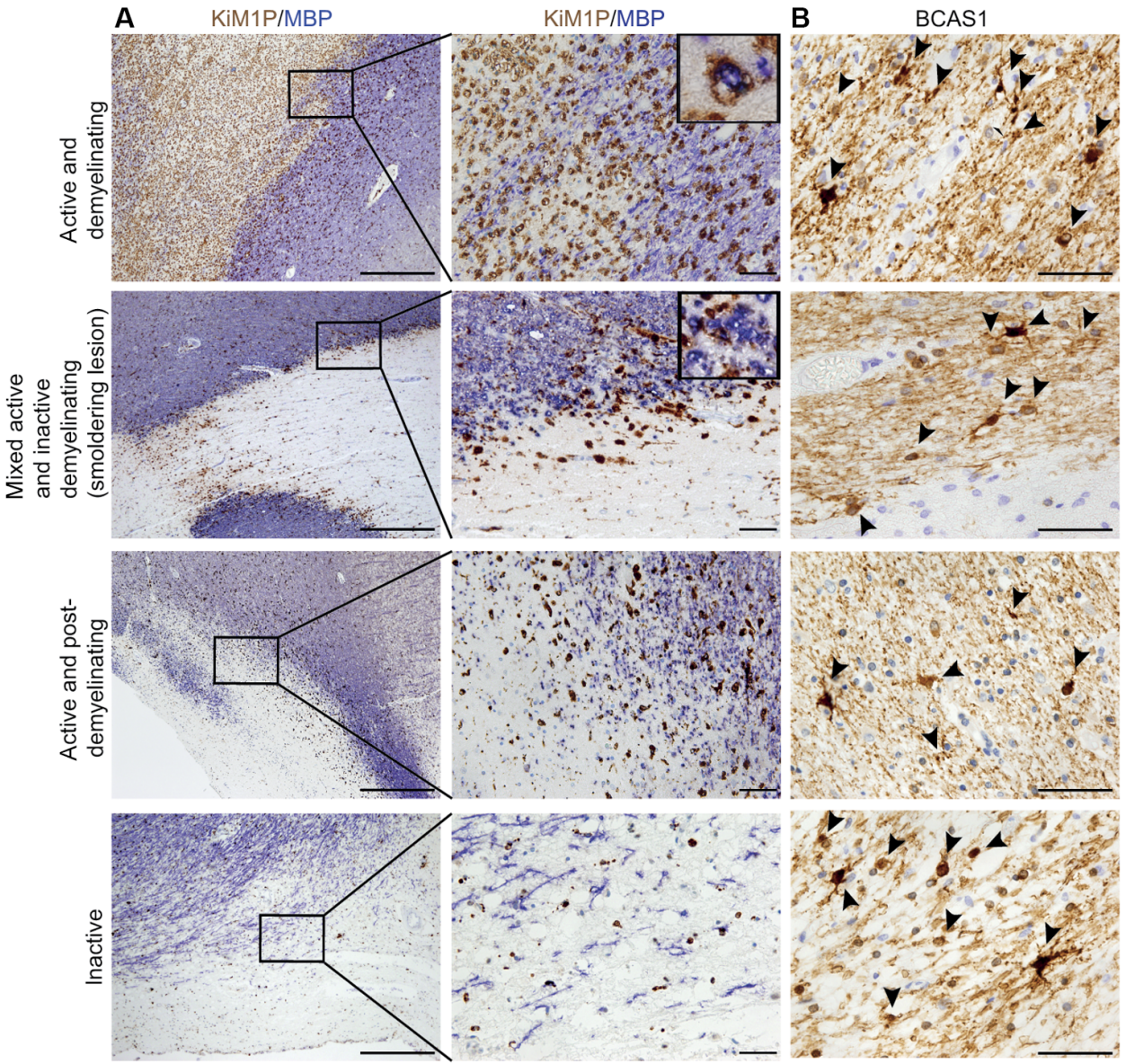

C

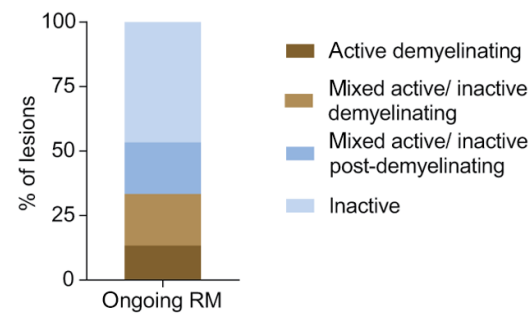

D

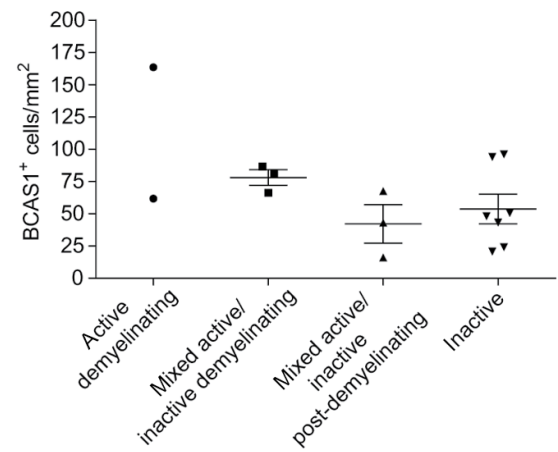

Fig. 8. Ongoing remyelination occurs independently from multiple sclerosis lesion activity. (A) Representative images showing the expression of the pan-macrophage/activated microglia marker KiM1P (brown) and MBP (blue) at the rim of multiple sclerosis lesions. Rows represent different inflammatory and demyelinating lesion activities according to Kuhlmann et al. (33). Insets in (A) highlight KiM1P ${ }^{+}$phagocytes containing $\mathrm{MBP}^{+}$particles. (B) Representative images showing BCAS1 expression at the rim of multiple sclerosis lesions of varying inflammatory and demyelinating activity; $\mathrm{BCAS}^{+}$oligodendrocytes are indicated by arrowheads. $\mathrm{BCAS} 1^{+}$oligodendrocytes extending multiple processes are observed at the lesion rim in all stages of lesion activity. (C) Percentage of lesions with different inflammatory and demyelinating activities containing areas of ongoing remyelination (ongoing RM) with BCAS1 ${ }^{+}$cells $(n=15)$. (D) Quantification of $\mathrm{BCAS}^{+}{ }^{+}$cell density in areas with ongoing remyelination of multiple sclerosis lesions of different lesion activities $(n=15)$. Quantitative data are means \pm SEM. Scale bars, $500 \mu \mathrm{m}$ (A, left column) and $50 \mu \mathrm{m}$ (all other images).

process of remyelination may operate on a different time scale and require additional cues in the human brain compared to the mouse brain (43). Thus, even if we unraveled the dynamic nature of $\mathrm{BCAS1}^{+}$oligo- dendrocytes by lineage tracing in mice, we cannot extrapolate these findings to humans. As a consequence, we do not know how long $\mathrm{BCAS1}^{+}$oligodendrocytes persist in the human brain. We found that $\mathrm{BCAS1}^{+}$ oligodendrocytes are formed in the fetal and newborn brain, largely disappear from the adult white matter, and are reformed in MS lesions. Yet, we lack information on their lifetime and cellular state in MS lesions. Whether $\mathrm{BCAS}^{+}$oligodendrocytes in humans represent newly formed or old cells locked in an intermediate state of differentiation cannot be determined to date. Nevertheless, the observation that $\mathrm{BCAS1}^{+}$ cells are associated with partially remyelinated lesions suggests that at least some of these cells are actively engaged in forming myelin. We envision that the identification of $\mathrm{BCAS1}^{+}$cells as an actively myelinating oligodendroglial cell population in humans that segregates from oligodendrocyte progenitor cells and mature oligodendrocytes will have profound implications for the functional analysis of myelination and remyelination in highly prevalent neuroinflammatory, neurodegenerative, and neuropsychiatric diseases. Furthermore, this work provides a conceptual and methodological basis for efforts to identify myelinregenerative compounds $(44,45)$.

\section{MATERIAL AND METHODS Study design}

The primary objective of this study was to develop molecular tools to distinguish the cellular subtypes or stages of the oligodendrocyte lineage. Immunohistochemistry and functional studies including lineage tracing and cell and protein turnover studies were performed in mice to establish $\mathrm{BCAS1}^{+}$cells as an oligodendroglial subpopulation. The formation of $\mathrm{BCAS1}^{+}$oligodendrocytes during remyelination was determined in two experimental models of demyelination and lysolecithin- and cuprizone-induced demyelination. Immunohistochemical studies were performed to associate $\mathrm{BCAS1}^{+}$cells with developmental myelination along the life span of a human brain. The brain tissue of patients with MS was examined to establish $\mathrm{BCAS1}^{+}$cells as a cellular marker of ongoing remyelination in the disease. The number of biological replicates $(n)$ per experiment is noted in each figure legend. The quantitative analyses were performed blinded. No outlier removal was performed. 


\section{Statistical analysis}

All statistics were calculated using the GraphPad Prism 6 software (GraphPad Software). The Kolmogorov-Smirnov test with DallalWilkinson-Lillie for $P$ value was carried out to test for normal distribution. To compare the two groups, a Student's two-tailed $t$ test or the Mann-Whitney test was applied. One-way analysis of variance (ANOVA) followed by Bonferroni's, Tukey's, or Newman-Keuls' post hoc test was performed for comparison of three and more than three groups, or one-way ANOVA with Kruskal-Wallis test with Dunn's multiple comparison tests was used as indicated in the figure legends. Data are means \pm SEM if not stated otherwise. Asterisks correspond to $P$ values of $<0.05\left(^{*}\right),<0.01(* *)$, and $\left.<0.001{ }^{* * *}\right)$.

\section{SUPPLEMENTARY MATERIALS}

www.sciencesignaling.org/cgi/content/full/9/419/eaam7816/DC1 Materials and Methods

Fig. S1. Validation of antiserum raised against BCAS1.

Fig. S2. BCAS1 specifically labels cells of oligodendrocyte lineage.

Fig. S3. Characterization of $\mathrm{BCAS1}^{+}$pre-mOLGs and mOLGs.

Fig. S4. Characterization of ENPP6 ${ }^{+}$oligodendrocytes in mice.

Fig. S5. BCAS1 is down-regulated in fully matured oligodendrocytes prepared from mice.

Fig. S6. Comparison of BCAS1 with 01 and 04 immunolabeling.

Fig. S7. BCAS1 is down-regulated in fully matured oligodendrocytes prepared from human iPSCs.

Fig. S8. Dynamics of $\mathrm{BCAS}^{+}$cells generation in adult mouse cortex.

Fig. S9. Quantification of the number of $\mathrm{BCAS1}^{+}$cells in the adult mouse brain.

Fig. S10. Developmentally born mOLGs continue myelination into late adulthood.

Fig. S11. BCAS1 ${ }^{+}$oligodendrocytes are distinct from $\mathrm{PLP}^{+}$and $\mathrm{ENPP}^{+}$oligodendrocytes in the human brain.

Fig. S12. Characterization of $\mathrm{BCAS1}^{+}$oligodendrocytes in the human brain.

Fig. S13. $\mathrm{BCAS}^{+}$oligodendroglia are rare in and around chronic demyelinated MS lesions and fully remyelinated shadow plaques.

Table S1. Clinical characteristics of patients to study the developmental expression of BCAS1. Table S2. Clinical characteristics of MS patients.

Table S3. Primary data.

References (46-48)

\section{REFERENCES AND NOTES}

1. N. Snaidero, W. Möbius, T. Czopka, L. H. P. Hekking, C. Mathisen, D. Verkleij, S. Goebbels, J. Edgar, D. Merkler, D. A. Lyons, K.-A. Nave, M. Simons, Myelin membrane wrapping of CNS axons by $\mathrm{PI}(3,4,5) \mathrm{P3}$-dependent polarized growth at the inner tongue. Cell 156, 277-290 (2014).

2. K.-A. Nave, H. B. Werner, Myelination of the nervous system: Mechanisms and functions. Annu. Rev. Cell Dev. Biol. 30, 503-533 (2014).

3. N. Baumann, D. Pham-Dinh, Biology of oligodendrocyte and myelin in the mammalian central nervous system. Physiol. Rev. 81, 871-927 (2001).

4. K.-J. Chang, S. A. Redmond, J. R. Chan, Remodeling myelination: Implications for mechanisms of neural plasticity. Nat. Neurosci. 19, 190-197 (2016).

5. H. Wake, P. R. Lee, R. D. Fields, Control of local protein synthesis and initial events in myelination by action potentials. Science 333, 1647-1651 (2011).

6. C. Demerens, B. Stankoff, M. Logak, P. Anglade, B. Allinquant, F. Couraud, B. Zalc, C. Lubetzki, Induction of myelination in the central nervous system by electrical activity. Proc. Natl. Acad. Sci. U.S.A. 93, 9887-9892 (1996).

7. E. G. Hughes, S. H. Kang, M. Fukaya, D. E. Bergles, Oligodendrocyte progenitors balance growth with self-repulsion to achieve homeostasis in the adult brain. Nat. Neurosci. 16, 668-676 (2013).

8. R. J. M. Franklin, C. Ffrench-Constant, Remyelination in the CNS: From biology to therapy. Nat. Rev. Neurosci. 9, 839-855 (2008).

9. L. Xiao, D. Ohayon, I. A. McKenzie, A. Sinclair-Wilson, J. L. Wright, A. D. Fudge, B. Emery, $\mathrm{H}$. Li and W. D. Richardson, Rapid production of new oligodendrocytes is required in the earliest stages of motor-skill learning. Nat. Neurosci. 19, 1210-1217 (2016).

10. K. M. Young, K. Psachoulia, R. B. Tripathi, S.-J. Dunn, L. Cossell, D. Attwell, K. Tohyama, W. D. Richardson, Oligodendrocyte dynamics in the healthy adult CNS: Evidence for myelin remodeling. Neuron 77, 873-885 (2013).

11. B. A. Barres, M. C. Raff, Proliferation of oligodendrocyte precursor cells depends on electrical activity in axons. Nature 361, 258-260 (1993).
12. I. A. McKenzie, D. Ohayon, H. Li, J. P. de Faria, B. Emery, K. Tohyama, W. D. Richardson, Motor skill learning requires active central myelination. Science 346, 318-322 (2014).

13. S. L. Bengtsson, Z. Nagy, S. Skare, L. Forsman, H. Forssberg, F. Ullén, Extensive piano practicing has regionally specific effects on white matter development. Nat. Neurosci. 8 , 1148-1150 (2005).

14. R. J. Zatorre, R. D. Fields, H. Johansen-Berg, Plasticity in gray and white: Neuroimaging changes in brain structure during learning. Nat. Neurosci. 15, 528-536 (2012).

15. M. S. Y. Yeung, S. Zdunek, O. Bergmann, S. Bernard, M. Salehpour, K. Alkass, S. Perl, J. Tisdale, G. Possnert, L. Brundin, H. Druid, J. Frisén, Dynamics of oligodendrocyte generation and myelination in the human brain. Cell 159, 766-774 (2014).

16. P. Patrikios, C. Stadelmann, A. Kutzelnigg, H. Rauschka, M. Schmidbauer, H. Laursen, P. S. Sorensen, W. Brück, C. Lucchinetti, H. Lassmann, Remyelination is extensive in a subset of multiple sclerosis patients. Brain 129, 3165-3172 (2006).

17. C. Collins, J. M. Rommens, D. Kowbel, T. Godfrey, M. Tanner, S.-I. Hwang, D. Polikoff, G. Nonet, J. Cochran, K. Myambo, K. E. Jay, J. Froula, T. Cloutier, W.-L. Kuo, P. Yaswen, S. Dairkee, J. Giovanola, G. B. Hutchinson, J. Isola, O.-P. Kallioniemi, M. Palazzolo, C. Martin, C. Ericsson, D. Pinkel, D. Albertson, W.-B. Li, J. W. Gray, Positional cloning of ZNF217 and $N A B C 1$ : Genes amplified at 20q13.2 and overexpressed in breast carcinoma. Proc. Natl. Acad. Sci. U.S.A. 95, 8703-8708 (1998).

18. Y. Zhang, K. Chen, S. A. Sloan, M. L. Bennett, A. R. Scholze, S. O'Keeffe, H. P. Phatnani, P. Guarnieri, C. Caneda, N. Ruderisch, S. Deng, S. A. Liddelow, C. Zhang, R. Daneman, T. Maniatis, B. A. Barres, J. Q. Wu, An RNA-sequencing transcriptome and splicing database of glia, neurons, and vascular cells of the cerebral cortex. J. Neurosci. 34, 11929-11947 (2014).

19. K. Ninomiya, T. Ishimoto, T. Taguchi, Subcellular localization of PMES-2 proteins regulated by their two cytoskeleton-associated domains. Cell. Mol. Neurobiol. 25, 899-911 (2005).

20. T. Ishimoto, K. Ninomiya, R. Inoue, M. Koike, Y. Uchiyama and H. Mori, Mice lacking BCAS1, a novel myelin-associated protein, display hypomyelination, schizophrenia-like abnormal behaviors, and upregulation of inflammatory genes in the brain. Glia 65, 727-739 (2017).

21. K. Sharma, S. Schmitt, C. G. Bergner, S. Tyanova, N. Kannaiyan, N. Manrique-Hoyos, K. Kongi, L. Cantuti, U.-K. Hanisch, M.-A. Philips, M. J. Rossner, M. Mann, M. Simons, Cell type- and brain region-resolved mouse brain proteome. Nat. Neurosci. 18, 1819-1831 (2015).

22. W. Huang, N. Zhao, X. Bai, K. Karram, J. Trotter, S. Goebbels, A. Scheller, F. Kirchhoff, Novel NG2-CreERT2 knock-in mice demonstrate heterogeneous differentiation potential of NG2 glia during development. Glia 62, 896-913 (2014).

23. L. Madisen, T. A. Zwingman, S. M. Sunkin, S. W. Oh, H. A. Zariwala, H. Gu, L. L. Ng, R. D. Palmiter, M. J. Hawrylycz, A. R. Jones, E. S. Lein, H. Zeng, A robust and high-throughput Cre reporting and characterization system for the whole mouse brain. Nat. Neurosci. 13, 133-140 (2010).

24. A. Chang, W. W. Tourtellotte, R. Rudick, B. D. Trapp, Premyelinating oligodendrocytes in chronic lesions of multiple sclerosis. N. Engl. J. Med. 346, 165-173 (2002).

25. B. D. Trapp, A. Nishiyama, D. Cheng, W. Macklin, Differentiation and death of premyelinating oligodendrocytes in developing rodent brain. J. Cell Biol. 137, 459-468 (1997).

26. N. Spassky, C. Olivier, I. Cobos, B. LeBras, C. Goujet-Zalc, S. Martínez, B. Zalc, J. L. Thomas, The early steps of oligodendrogenesis: Insights from the study of the plp lineage in the brain of chicks and rodents. Dev. Neurosci. 23, 318-326 (2001).

27. G. S. Tomassy, D. R. Berger, H.-H. Chen, N. Kasthuri, K. J. Hayworth, A. Vercelli, H. S. Seung, J. W. Lichtman, P. Arlotta, Distinct profiles of myelin distribution along single axons of pyramidal neurons in the neocortex. Science 344, 319-324 (2014).

28. M. N. Rasband, E. Peles, The nodes of Ranvier: Molecular assembly and maintenance. Cold Spring Harb. Perspect. Biol. 8, a020495 (2016).

29. M. Ehrlich, S. Mozafari, M. Glatza, L. Starost, S. Velychko, A.-L. Hallmann, Q.-L. Cui, A. Schambach, K.-P. Kim, C. Bachelin, A. Marteyn, G. Hargus, R. M. Johnson, J. Antel, J. Sterneckert, H. Zaehres, H. R. Schöler, A. Baron-Van Evercooren, T. Kuhlmann, Rapid and efficient generation of oligodendrocytes from human induced pluripotent stem cells using transcription factors. Proc. Natl. Acad. Sci. U.S.A. 114, E2243-E2252 (2017).

30. S. Schneider, A. Gruart, S. Grade, Y. Zhang, S. Kröger, F. Kirchhoff, G. Eichele, J. M. Delgado García, L. Dimou, Decrease in newly generated oligodendrocytes leads to motor dysfunctions and changed myelin structures that can be rescued by transplanted cells. Glia 64, 2201-2218 (2016).

31. G. Whelan, E. Kreidl, G. Wutz, A. Egner, J.-M. Peters and G. Eichele, Cohesin acetyltransferase $\mathrm{EscO} 2$ is a cell viability factor and is required for cohesion in pericentric heterochromatin. EMBO J. 31, 71-82 (2012).

32. M. Krüger, M. Moser, S. Ussar, I. Thievessen, C. A. Luber, F. Forner, S. Schmidt, S. Zanivan, R. Fässler, M. Mann, SILAC mouse for quantitative proteomics uncovers kindlin-3 as an essential factor for red blood cell function. Cell 134, 353-364 (2008).

33. T. Kuhlmann, S. Ludwin, A. Prat, J. Antel, W. Brück, H. Lassmann, An updated histological classification system for multiple sclerosis lesions. Acta Neuropathol. 133, 13-24 (2016).

34. J. W. Prineas, R. O. Barnard, E. E. Kwon, L. R. Sharer, E. S. Cho, Multiple sclerosis: Remyelination of nascent lesions. Ann. Neurol. 33, 137-151 (1993). 
35. S. Marques, A. Zeisel, S. Codeluppi, D. van Bruggen, A. Mendanha Falcão, L. Xiao, H. Li, M. Häring, H. Hochgerner, R. A. Romanov, D. Gyllborg, A. B. Muñoz-Manchado, G. La Manno, P. Lönnerberg, E. M. Floriddia, F. Rezayee, P. Ernfors, E. Arenas, J. Hjerling-Leffler, T. Harkany, W. D. Richardson, S. Linnarsson, G. Castelo-Branco, Oligodendrocyte heterogeneity in the mouse juvenile and adult central nervous system. Science 352, 1326-1329 (2016).

36. A. Zeisel, A. B. Muñoz-Manchado, S. Codeluppi, P. Lönnerberg, G. La Manno, A. Juréus, S. Marques, H. Munguba, L. He, C. Betsholtz, C. Rolny, G. Castelo-Branco, J. Hjerling-Leffler and S. Linnarsson, Cell types in the mouse cortex and hippocampus revealed by single-cell RNA-seq. Science 347, 1138-1142 (2015).

37. M. R. Freeman, D. H. Rowitch, Evolving concepts of gliogenesis: A look way back and ahead to the next 25 years. Neuron 80, 613-623 (2013).

38. B. Emery, Regulation of oligodendrocyte differentiation and myelination. Science $\mathbf{3 3 0}$ 779-782 (2010).

39. S. A. Goldman, N. J. Kuypers, How to make an oligodendrocyte. Development 142, 3983-3995 (2015).

40. S. A. Back, N. L. Luo, N. S. Borenstein, J. M. Levine, J. J. Volpe, H. C. Kinney, Late oligodendrocyte progenitors coincide with the developmental window of vulnerability for human perinatal white matter injury. J. Neurosci. 21, 1302-1312 (2001).

41. J. M. Ruckh, J.-W. Zhao, J. L. Shadrach, P. van Wijngaarden, T. N. Rao, A. J. Wagers, R. J. M. Franklin, Rejuvenation of regeneration in the aging central nervous system. Cell Stem Cell 10, 96-103 (2012).

42. F. J. Sim, C. R. McClain, S. J. Schanz, T. L. Protack, M. S. Windrem, S. A. Goldman, CD140a identifies a population of highly myelinogenic, migration-competent and efficiently engrafting human oligodendrocyte progenitor cells. Nat. Biotechnol. 29, 934-941 (2011).

43. D. Buchet, C. Garcia, C. Deboux, B. Nait-Oumesmar, A. Baron-Van Evercooren, Human neural progenitors from different foetal forebrain regions remyelinate the adult mouse spinal cord. Brain 134, 1168-1183 (2011).

44. F. J. Najm, M. Madhavan, A. Zaremba, E. Shick, R. T. Karl, D. C. Factor, T. E. Miller, Z. S. Nevin, C. Kantor, A. Sargent, K. L. Quick, D. M. Schlatzer, H. Tang, R. Papoian, K. R. Brimacombe, M. Shen, M. B. Boxer, A. Jadhav, A. P. Robinson, J. R. Podojil, S. D. Miller, R. H. Miller, P. J. Tesar, Drug-based modulation of endogenous stem cells promotes functional remyelination in vivo. Nature 522, 216-220 (2015).

45. F. Mei, S. P. J. Fancy, Y.-A. A. Shen, J. Niu, C. Zhao, B. Presley, E. Miao, S. Lee, S. R. Mayoral, S. A. Redmond, A. Etxeberria, L. Xiao, R. J. M. Franklin, A. Green, S. L. Hauser, J. R. Chan, Micropillar arrays as a high-throughput screening platform for therapeutics in multiple sclerosis. Nat. Med. 20, 954-960 (2014).

46. D. P. Leone, S. Genoud, S. Atanasoski, R. Grausenburger, P. Berger, D. Metzger, W. B. Macklin, P. Chambon, U. Suter, Tamoxifen-inducible glia-specific Cre mice for somatic mutagenesis in oligodendrocytes and Schwann cells. Mol. Cell. Neurosci. 22, 430-440 (2003).
47. T. Nakamura, M. C. Colbert, J. Robbins, Neural crest cells retain multipotential characteristics in the developing valves and label the cardiac conduction system. Circ. Res. 98, 1547-1554 (2006).

48. C. Simon, H. Lickert, M. Götz, L. Dimou, Sox10-iCreER ${ }^{T 2}$ : A mouse line to inducibly trace the neural crest and oligodendrocyte lineage. Genesis 50, 506-515 (2012).

Acknowledgments: We thank G. Schulz, K. Schulz, and O. Kowatsch for their expert technical assistance. We also thank G. Eichele (Max Planck Institute, Göttingen, Germany) for Esco2fl/fl mice, B. Zalc (INSERM, Paris, France) for PLP-G mice, and F. Kirchhoff (University of Saarland, Homburg, Germany) for NG2-CreERT2 mice, and we are grateful for being able to use PLP-CreERT2 mice in collaboration with U. Suter (ETH Zürich, Switzerland). Funding: This work was supported by an ERC CoG grant (M.S.) and grants from the German Research Foundation (SPP128, SPP1757, SFB1286, and TRR43), the Tschira-Stiftung and the Adelson Foundation (M.S.), the Cluster of Excellence and DFG Research SyNergy (M.S. and L.D.), the Hertie-Stiftung, and the German Multiple Sclerosis Society (C. Stadelmann and W.B.). Author contributions: M.S., C. Stadelmann, M.K.F., and F.v.d.M. designed the research and analyzed the data. M.K.F. performed mouse in vitro and in vivo experiments, EdU and SILAC pulse labeling, morphological analysis, and characterization of oligodendroglial subpopulations in mice. F.v.d.M. performed the experiments using human samples. C. Sergiou, V.S., and C.W. performed remyelination experiments in mice. S.S. generated Bcas1 knockout mice. S.M. and E.F.F. performed and analyzed the mass spectrometry data. M.E. and L.S. performed the experiments using human iPSCS. P.S. and L.C.-C. performed immunohistochemistry. T.K. supervised the experiments using human iPSCs. H.U. supervised mass spectrometry analysis. S.J. and L.D. performed the experiments with Esco2 mice. W.B. provided the human samples. M.S. wrote the paper. C. Stadelmann, M.K.F., F.v.d.M., L.D., W.B., H.U., and L.D. edited the manuscript. Competing interests: The authors declare that they have no competing interests. Data and materials availability: All data are contained within the manuscript and the Supplementary Materials.

Submitted 16 January 2017 Resubmitted 13 July 2017 Accepted 14 November 2017 Published 6 December 2017 10.1126/scitransImed.aam7816

Citation: M. K. Fard, F. van der Meer, P. Sánchez, L. Cantuti-Castelvetri, S. Mandad, S. Jäkel, E. F. Fornasiero, S. Schmitt, M. Ehrlich, L. Starost, T. Kuhlmann, C. Sergiou, V. Schultz, C. Wrzos, W. Brück, H. Urlaub, L. Dimou, C. Stadelmann, M. Simons, BCAS1 expression defines a population of early myelinating oligodendrocytes in multiple sclerosis lesions. Sci. Transl. Med. 9, eaam7816 (2017). 


\section{Science Translational Medicine}

\section{BCAS1 expression defines a population of early myelinating oligodendrocytes in multiple sclerosis lesions}

Maryam K. Fard, Franziska van der Meer, Paula Sánchez, Ludovico Cantuti-Castelvetri, Sunit Mandad, Sarah Jäkel, Eugenio F. Fornasiero, Sebastian Schmitt, Marc Ehrlich, Laura Starost, Tanja Kuhlmann, Christina Sergiou, Verena Schultz, Claudia Wrzos, Wolfgang Brück, Henning Urlaub, Leda Dimou, Christine Stadelmann and Mikael Simons

Sci Transl Med 9, eaam7816.

DOI: 10.1126/scitranslmed.aam7816

\section{Mapping active myelination with BCAS1}

Neuronal axon demyelination causes motor and cognitive impairments in multiple sclerosis (MS) and other demyelinating disorders. Although remyelinating strategies have been proposed, the lack of markers to detect areas of active myelination hampers the development of effective therapies. Fard et al. show that myelinating oligodendrocytes constitute a unique population expressing breast carcinoma amplified sequence 1 (BCAS1) in rodent and human brain tissue. In brain samples from deceased MS patients, BCAS1 ${ }^{+}$cells are present around lesions, suggesting that remyelination might occur during MS and that BCAS1 expression could be used to track responses to remyelinating compounds for treating demyelinating disorders.

ARTICLE TOOLS

SUPPLEMENTARY MATERIALS

RELATED

CONTENT

REFERENCES

PERMISSIONS http://stm.sciencemag.org/content/9/419/eaam7816

http://stm.sciencemag.org/content/suppl/2017/12/04/9.419.eaam7816.DC1

http://stm.sciencemag.org/content/scitransmed/8/364/364ra152.full http://stm.sciencemag.org/content/scitransmed/8/362/362ra146.full http://stm.sciencemag.org/content/scitransmed/7/310/310ra166.full

This article cites 48 articles, 17 of which you can access for free http://stm.sciencemag.org/content/9/419/eaam7816\#BIBL

http://www.sciencemag.org/help/reprints-and-permissions

Use of this article is subject to the Terms of Service

Science Translational Medicine (ISSN 1946-6242) is published by the American Association for the Advancement of Science, 1200 New York Avenue NW, Washington, DC 20005. 2017 (C The Authors, some rights reserved; exclusive licensee American Association for the Advancement of Science. No claim to original U.S. Government Works. The title Science Translational Medicine is a registered trademark of AAAS. 